\title{
Generalization of Fear Inhibition by Disrupting Hippocampal Protein Synthesis-Dependent Reconsolidation Process
}

\author{
Chih-Hao Yang', Chiung-Chun Huang' and Kuei-Sen Hsu*,' \\ 'Department of Pharmacology, College of Medicine, National Cheng Kung University, Tainan, Taiwan
}

\begin{abstract}
Repetitive replay of fear memories may precipitate the occurrence of post-traumatic stress disorder and other anxiety disorders. Hence, the suppression of fear memory retrieval may help prevent and treat these disorders. The formation of fear memories is often linked to multiple environmental cues and these interconnected cues may act as reminders for the recall of traumatic experiences. However, as a convenience, a simple paradigm of one cue pairing with the aversive stimulus is usually used in studies of fear conditioning in animals. Here, we built a more complex fear conditioning model by presenting several environmental stimuli during fear conditioning and characterize the effectiveness of extinction training and the disruption of reconsolidation process on the expression of learned fear responses. We demonstrate that extinction training with a single-paired cue resulted in cue-specific attenuation of fear responses but responses to other cures were unchanged. The cue-specific nature of the extinction persisted despite training sessions combined with D-cycloserine treatment reveals a significant weakness in extinction-based treatment. In contrast, the inhibition of the dorsal hippocampus $(\mathrm{DH})$ but not the basolateral amygdala (BLA)-dependent memory reconsolidation process using either protein synthesis inhibitors or genetic disruption of CAMP-response-element-binding protein-mediated transcription comprehensively disrupted the learned connections between fear responses and all paired environmental cues. These findings emphasize the distinct role of the $\mathrm{DH}$ and the BLA in the reconsolidation process of fear memories and further indicate that the disruption of memory reconsolidation process in the $\mathrm{DH}$ may result in generalization of fear inhibition.

Neuropsychopharmacology (20II) 36, 1992-2008; doi:I0.1038/npp.20I I.87; published online I8 May 20II
\end{abstract}

Keywords: stress; fear memory; extinction; reconsolidation; hippocampus; amygdala

\section{INTRODUCTION}

Aversive emotional memories of previously traumatic events could be an important predisposing factor affecting the precipitation of relapse of post-traumatic stress disorder (PTSD) and other anxiety disorders. Since the fearful experiences could be easily retrieved by the conditioned cues linked with traumatic events, it is now widely assumed that the removal of cue-induced conditioned fear responses would be supremely helpful for extinguishing fear memories (Schafe et al, 2001; Ressler and Mayberg, 2007; Radulovic and Tronson, 2010). One successful strategy for weakening the association between the conditioned fear memory with its connected cues is referred to as extinction training (the repeated exposure to the conditioned stimulus (CS) in the absence of the reinforcing unconditioned stimulus (US) with which it was paired previously, the conditioned responses elicited by the CS dissipate over

*Correspondence: Dr K-S Hsu, Department of Pharmacology, College of Medicine, National Cheng Kung University, No. I, University Road, Tainan 70I, Taiwan, Tel: + 88662353535 ext. 5498, Fax: + 8866274 9296, E-mail: richard@mail.ncku.edu.tw

Received 18 December 2010; revised 25 March 201।; accepted 20 April 2011 time). Extinction-based therapeutic approach was first used by Pavlov (1927) and Bouton (2002) and has been clinically used for years to treat fear- and anxiety-related disorders such as phobias and PTSD (Stein and Matsunaga, 2006; Kushner et al, 2007). Although extinction-based exposure therapy has been proven effective in some cases, not everyone benefits and many responders relapse due to spontaneous fear recovery, reinstatement, or renewal (Rescorla and Heth, 1975; Dirikx et al, 2004; Hermans et al, 2006; Vansteenwegen et al, 2006; Schiller et al, 2008). Recently, D-cycloserine (DCS; a partial agonist of the $\mathrm{N}$-methyl-D-aspartate receptor) has been used to strengthen the experimental extinction of learned fear (Walker et al, 2002; Ressler et al, 2004; Ledgerwood et al, 2005) and it has been shown to suppress the reinstatement of fear effectively in both rodents and humans.

Unfortunately, there is still a high relapse rate of distressing symptoms after apparently successful extinction treatment even in combination with DCS (Kushner et al, 2007; Schiller et al, 2008). While the neurobiological basis of failure to prevent the return of extinguished conditioned responses following extinction therapy remains elusive, possible causes include the highly cue-specific nature of extinction training (Bouton and King, 1983; Kearns and Weiss, 2007). Indeed, traumatic fear memories are rarely 
formed under single-cue condition. Traumatic fear memories always come along with multiple environmental cues (eg, sounds, odors, tastes, feelings, and context) around and all these informative units incorporate into the fearful memory episodic network once the memory had been consolidated. Once fear memory is established, any of the cues linked to the traumatic events may be able to fully recall fear responses. Hence, research effort is needed to develop alternative strategies or more effective ways to strengthen extinction training.

Another potential strategy for reducing the impact of conditioned cues is to disrupt the memory reconsolidation process. Numerous studies have shown that previously consolidated memory can transiently return to a labile status after reactivation and then undergoing a protein synthesis-dependent reconsolidation process (Nader et al, 2000a; Nader and Hardt, 2009). The disruption of memory reconsolidation process has been seized upon as a potential therapeutic approach for the treatment of PTSD and anxiety disorders (Debiec et al, 2002; Kindt et al, 2009; Monfils et al, 2009; Schiller et al, 2010). Converging evidence from animal and human studies have revealed that the disruption of the reconsolidation process can effectively erase fear memories and prevent the return of fear (Nader et al, 2000b; Kindt et al, 2009; Schiller et al, 2010). Although the molecular mechanisms of memory reconsolidation remain largely unknown, it is generally believed that de novo protein synthesis and increased release of norepinephrine are mandatory (Nader et al, 2000b; Tronson and Taylor, 2007; Kindt et al, 2009).

For convenience, a single CS-US pairing paradigm has been widely employed for studying the mechanisms by which fear is acquired in animals. As mentioned earlier, clinical experience, however, suggests that the formation of fear memories is often triggered by multiple interconnected environmental cues associated with traumatic events. To date, there has been little experimental analysis of the acquisition of conditioned responses with multiple cues in animals. In the present study, we have therefore sought to develop a more complex fear conditioning model using multiple environmental cues (simultaneously presented during the training session) and investigate the effectiveness of extinction training and the disruption of memory reconsolidation process on the expression of learned fear responses.

\section{MATERIALS AND METHODS}

\section{Animals}

Adult male Sprague-Dawley rats (250-300 g) were used in all studies. Rats were housed in groups of three in a humidityand temperature-controlled $\left(25 \pm 1^{\circ} \mathrm{C}\right)$ vivarium on a 12 -h light/dark cycle (lights on at 0600 hours), with access to food and water ad libitum. Rats were acclimated in the animal research facility for at least 7 days before use in behavioral experiments. All experiments were conducted during the light phase of the cycle. All procedures were conducted in accordance with the National Institutes of Health Guide for the Care and Use of Laboratory Animals (NIH Publication No. 80-23, revised 1996) and were approved by the Institutional Animal Care and Use
Committee of National Cheng Kung University. All efforts were made to minimize the number of animals used and their suffering.

\section{Fear Conditioning Procedure}

Rats were initially habituated to the conditioning context for $10 \mathrm{~min}$ each day for 3 days without odor, tone, and visual cues presented. Twenty-four hours later, rats were concomitantly conditioned with three different environmental cues (CS: odor, tone, and visual context) paired with electric tail shock (US, 60 pairings). During the conditioning period, rat was placed into a cylinder-shaped restrainer and the restrainer was placed in the center of a Plexiglas conditioning chamber $\left(40 \times 25 \times 25 \mathrm{~cm}^{3}\right)$ that was enclosed within a sound attenuating cubicle (Model ENV-018V; MED Associates, St Albans, VT). Numerous visual cues including colored star-, moon-, and cross-shaped marks were attached on the walls of the conditioning chamber to provide contextual visual cues. The chamber was sprayed regularly with $0.1 \%$ Citrus bergamia (Primavera Life, Sulzberg, Germany) as an odor cue and a tone cue $(2 \mathrm{kHz}$ at $80 \mathrm{~dB})$ was delivered $1 \mathrm{~s}$ before the tail shock $(1 \mathrm{~mA}, 30-$ $90 \mathrm{~s}$ apart) through a speaker attached to the wall of the chamber. The conditioning chamber was cleaned with $75 \%$ alcohol after each use.

\section{Cannulation and Drug Treatment}

To regional delivery of protein synthesis inhibitors into the basolateral amygdala (BLA) or the CA1 region of the dorsal hippocampus (DH), rats were deeply anesthetized with pentobarbital $(50 \mathrm{mg} / \mathrm{kg}$, intraperitoneal injection) for cannulas implantation, and 22 gauge cannulas were stereotaxically placed directed toward the stratum pyramidale of the CA1 region of the $\mathrm{DH}$ (coordinates: anterioposterior $=-3.8 \mathrm{~mm}$ from $\quad$ bregma; $\quad$ lateral $= \pm 3 \mathrm{~mm}$; ventral $=2.8 \mathrm{~mm}$ ) or toward the BLA (coordinates: anterioposterior $=-2.5 \mathrm{~mm}$ from bregma; lateral $= \pm 5.3 \mathrm{~mm}$; ventral $=7.8 \mathrm{~mm}$ ), in accordance with the description by Paxinos and Watson (2007). The cannulas were fixed to the skull with dental cement. The animals were allowed to recover from surgery for at least 7 days before the experiments started. Anisomycin (Sigma-Aldrich, St Louis, MO) was dissolved in equimolar $\mathrm{HCl}$, diluted with aCSF and adjusted to $\mathrm{pH} 7.4$ with $\mathrm{NaOH}$. Cycloheximide (Sigma-Aldrich) or anisomycin was bilaterally injected into the both side of hippocampus or amygdala at indicated time point after memory retrieval by using an infusion pump (CAM/100; CAM Microdialysis, Solna, Sweden). A total volume of $1 \mu \mathrm{l}$ was infused into each side over $2 \mathrm{~min}$, and the infusion cannulae was kept in place for an additional 3 min to reduce the backflow of the injectant. Cannula placement was verified post hoc in all rats by injection of methylene blue. Rats with injection cannula placements outside the BLA or the $\mathrm{DH}$ or with extensive tissue damage at the injection needle site were excluded from the analyses. After this, they were placed back to their home cage for 3 days before the commencement of behavioral testing. 


\section{Construction and Production of Engineered Lentiviruses}

Engineered self-inactivating recombinant lentiviruses were used for stably in vivo genetic targeting to intervene cAMPresponse-element-binding protein (CREB)-mediated transcriptional activity. All viruses were produced by cotransfection of lentiviral DNA with two helper plasmids: vesicular stomatitis virus envelope glycoprotein (VSV-G) and $\Delta 8.9$ in HEK293T cells (Kutner et al, 2009). Medium containing recombinant lentiviruses were harvested $36-48 \mathrm{~h}$ after transfection and ultracentrifuged to get the concentrated lentiviral particles. The pellets were then resuspended by phosphate buffer solution with titers of $10^{8} \sim 10^{9}$ units $/ \mathrm{ml}$. For the cloning of the dominant-negative CREB (KCREB) construct (Walton et al, 1992), a mutant variant of CREB protein that contains mutations in its DNA-binding domain was first cloned to fuse with the enhanced green fluorescence protein (EGFP) gene and then the KCREB-EGFP was further excised and inserted into a lentiviral backbone under the control of CAG promoter.

\section{Microinjection of Lentiviral Vectors}

Recombinant lentiviruses expressing the KCREB or EGFP were injected bilaterally into the BLA or the CA1 region of the $\mathrm{DH}$ using standard stereotaxic procedures. Under pentobarbital anesthesia, concentrated virus-stock solution was injected into the targeted sites $(0.5 \mu \mathrm{l}$ per site at $0.25 \mu \mathrm{l} / \mathrm{min}$ ) by using a Hamilton syringe with the 34-gauge blunted-tip needle. All rats received four different sites of viral injection targeting the CA1 region of the $\mathrm{DH}$ (coordinates: anterioposterior $=-3.8 \mathrm{~mm}$ from bregma; lateral $= \pm 3 \mathrm{~mm}$; ventral $=3 \mathrm{~mm}$; anterioposterior $=-4.5 \mathrm{~mm}$ from bregma; lateral $= \pm 3.5 \mathrm{~mm}$; ventral $=3.2 \mathrm{~mm}$ ) or the BLA (coordinates: anterioposterior $=-2.5 \mathrm{~mm}$ from bregma; lateral $= \pm 5.0 \mathrm{~mm} ; \quad$ ventral $=7.8 \mathrm{~mm} ; \quad$ anterioposterior $=$ $-3.5 \mathrm{~mm}$ from bregma; lateral $= \pm 5.3 \mathrm{~mm}$; ventral $=$ $7.8 \mathrm{~mm}$ ), in accordance with the description by Paxinos and Watson (2007). Rats were allowed to recover for 7 days before the memory trace reactivation.

\section{Plasma Corticosterone Assay}

Blood samples were obtained from the tail just before and immediately after stress, centrifuged at $1000 \times \mathrm{g}$ and plasma was separated and stored at $-20^{\circ} \mathrm{C}$. Plasma corticosterone levels were determined using a commercially available enzyme immunoassay (EIA) kit (Cayman Chemical, Ann Arbor, MI) according to the manufacturer's instructions as previously described (Yang et al, 2004). All assays were carried out in duplicate. The detection limit was $24 \mathrm{pg} / \mathrm{ml}$. Intra- and inter-assay variations were 4.1 and $9.5 \%$, respectively.

\section{Odor Preference Test}

An odor preference test was used to evaluate the formation of associative memory with the conditioned olfactory cue. The test apparatus consisted of a square chamber with ground area measuring $40 \times 40 \mathrm{~cm}^{2}$, and object preference test were conducted with dimmed light $(\sim 10$ Lux $)$. Test consists of four sessions for 2 consecutive days. Rats were first accumulated to the testing chamber for $10 \mathrm{~min}$ without any objects, and then returned to their home cages. Twentyfour hours after the training session, rats were first tested in the chamber for two identical objects without any odor sprayed on the objects. Rats with a strong bias to the objects or obvious defects in the exploration motivation for the objects were excluded from the test. After 2 or $3 \mathrm{~h}$, rats received a second test for two identical objects but one of them was sprayed with the odor cue (paired or unpaired, randomly) and $2-3 \mathrm{~h}$ later, rats were re-exposed to the chamber to examine the odor object preference. The durations for the rats spent in exploring the objects were video recorded using a digital video camera and scoring was performed with the behavioral tracking system Ethovision (Noldus, The Netherlands). The behavioral preference score were evaluated using: (1) percentage preference on the odor object (time spent on the odor object/time spent on the odored object + time spent on the non-odor object) $\times 100 \%$ and (2) difference in the number of exploration (total number exploring the odor object-total number exploring the non-odor object).

\section{Freezing Behaviors Induced by Tone Cue}

Tone-induced freezing behaviors were used to reflect the retrieval of fear memories by the conditioned auditory cue. The fear responses were detected using a computercontrolled context conditioning system (MED Associates), which consists of a testing chamber $\left(30 \times 24 \times 24 \mathrm{~cm}^{3}\right)$, with a digital near infrared camera recording the animals' behaviors from the ceiling of the chamber. There were two phases during the behavioral test. In the first phase, freezing was recorded for $3 \mathrm{~min}$ without the conditioned tone cue $(2 \mathrm{kHz}$ at $80 \mathrm{~dB})$ presentation. In the second phase, the tone cue was turned on and freezing was recorded for another $3 \mathrm{~min}$. To test if the animals display specific fear memories to the conditioned tone, the freezing responses to an unconditioned tone $(20 \mathrm{kHz}$ at $80 \mathrm{~dB})$ were also tested. The behavior data were analyzed by differential subtraction of two consecutive images captured at $7.5 \mathrm{~Hz}$ to calculate the significant motion pixels (SMP). A freezing behavior was defined as the value of SMP $<20$ at any indicated time point.

\section{Conditioned Place Aversion Test}

The conditioned place aversion (CPA) test was performed with an apparatus $\left(25 \times 50 \times 25 \mathrm{~cm}^{3}\right)$ consisted of two rectangular chambers, separated by guillotine door. On the day of CPA testing, the rats were placed in the apparatus and allowed free access to two chambers for $10 \mathrm{~min}$ without tone presentation. After this, the door was closed and the rats were restricted in one chamber pairing with the conditioned tone presentation. Rats were first placed in the left chamber (white floor) for $3 \mathrm{~min}$ without tone presentation and then rats were placed into the right chamber (black/white striped floor) with a paired tone cue presentation for $3 \mathrm{~min}$. After the training session, rats were placed back into the left chamber with tone turning off again. Following three rounds of OFF/ON tone training, rats were returned to their home cages. The CPA behaviors were 
analyzed $1 \mathrm{~h}$ later without any tone cue presentation in the chambers. The percentage of time spent in the tone-paired chamber and the escape latency to the tone-paired chamber were analyzed. To test if the animals display specific fear memories to the conditioned tone, the avoidance behaviors to an unconditioned tone $(20 \mathrm{kHz}$ at $80 \mathrm{~dB})$ were also tested.

\section{Extinction Training}

Two different types of extinction training protocols were used in this study. In the first protocol, a standard extinction training over three consecutive days was used. Rats were placed in a novel chamber with conditioned tone cue presentation (10 trains of $30 \mathrm{~s}$ tone with an inter-train interval of $5 \mathrm{~s}$ ). All rats received a single session of extinction training every day between 1100 and 1400 hours. In the second protocol, rats were intraperitoneally injected with DCS $(30 \mathrm{mg} / \mathrm{kg}$; Sigma-Aldrich) $30 \mathrm{~min}$ before the standard extinction training for 3 consecutive days. For the extinction training with a single olfactory cue, rats were placed in a novel chamber with conditioned odor cue presentation for $30 \mathrm{~min}$ per day over 3 consecutive days.

\section{Aggressive Behavior Test}

A resident-intruder aggressive behavior test was used to evaluate the retrieval of fear memories by the conditioned visual context cue. In the test session, the testing rats were placed into the conditioned chamber for $10 \mathrm{~min}$ and then shortly an experimentally naive intruder was placed in the chamber. The behaviors of the testing rats spent kicking, biting, fight, and clinching in $5 \mathrm{~min}$ with the intruder were recorded. The latency to the first attack and the total duration of attack were determined.

\section{Contextual Fear Conditioning}

The fear conditioning experiments were performed as described previously (Radulovic et al, 1998; Chang et al, 2009) using a computer-controlled context conditioning system (Med Associates). In brief, a rat was placed into the conditioning chamber $\left(30 \times 24 \times 24 \mathrm{~cm}^{3}\right)$ and allowed to explore for $3 \mathrm{~min}$ followed by five aversive electrical footshocks ( $3 \mathrm{~s}, 0.6 \mathrm{~mA}$ with $30 \mathrm{~s}$ inter-shock interval) through a stainless steel grid floor. After the last shock, animals were allowed to explore the context for additional 2 min before return to their home cages. The behavior of the animals was recorded using a digital near infrared video camera on the ceiling of the sound attenuating cubicle. Context-dependent freezing responses were measured immediately, 1 , or $24 \mathrm{~h}$ after fear conditioning. The freezing response was scored as the total time the rat spent in freezing during the 3-min test session.

\section{Western Blotting}

The microdissected DH CA1 tissue samples were transferred into ice-cold lysis buffer ( $\mathrm{pH}$ 7.4) containing a cocktail of protein phosphatase and proteinase inhibitors ( $50 \mathrm{mM}$ Tris- $\mathrm{HCl}, 100 \mathrm{mM} \mathrm{NaCl}, 15 \mathrm{mM}$ sodium pyrophosphate, $50 \mathrm{mM}$ sodium fluoride, $1 \mathrm{mM}$ sodium orthovanadate, $5 \mathrm{mM}$ EGTA, $5 \mathrm{mM}$ EDTA, $1 \mathrm{mM}$ phenylmethylsulfonyl fluoride, $1 \mu \mathrm{M}$ microcystin-LR, $1 \mu \mathrm{M}$ okadaic acid, $0.5 \%$ Triton X-100, $2 \mathrm{mM}$ benzamidine, $60 \mu \mathrm{g} / \mathrm{ml}$ aprotinin, and $60 \mu \mathrm{g} / \mathrm{ml}$ leupeptin) to avoid dephosphorylation and degradation of proteins, and ground with a pellet pestle (Kontes glassware, Vineland, NJ). Samples were sonicated and spun down at $15000 \mathrm{~g}$ at $4^{\circ} \mathrm{C}$ for $10 \mathrm{~min}$. The supernatant was then assayed for total protein concentration using Bio-Rad Bradford Protein Assay Kit (Hercules, CA). The proteins in each sample were electrophoretically separated in a 7.5\% SDS-PAGE gel. After the transfer on nitrocellulose membranes, blots were blocked in TBS containing $3 \%$ bovine serum albumin and $0.01 \%$ Tween 20 for $1 \mathrm{~h}$ and then blotted overnight at $4{ }^{\circ} \mathrm{C}$ with antibody that recognize c-fos $(1: 500$; Santa Cruz Biotechnology, Santa Cruz, CA). It was then probed with HRP-conjugated secondary antibody for $1 \mathrm{~h}$ and developed using the ECL immunoblotting detection system. Immunoblots were analyzed by densitometry using Bio-Profil BioLight PC software.

\section{Open-Field Test}

For the open-field test, rats were placed individually in the center of a test chamber to freely explore for 5 min under a low illumination $(\sim 10 \mathrm{Lux})$. The test chamber consisted of a circular floor $(50 \mathrm{~cm}$ in diameter), with a $40-\mathrm{cm}$ high wall set on a non-reflective black plastic base. The behavior of the animals was video recorded using a digital video camera and scoring was performed with the behavioral tracking system Ethovision (Nodus). The activity was evaluated based on the number of entries into the central zone, time spent in central zone, and total distance traveled in the open field. After each trial, the apparatus was thoroughly cleaned with $40 \%$ ethanol. The percentage of time spent in the center zone is defined as the percentage of time for the animals exploring the central $25 \%$ (25 cm in diameter) of the chamber.

\section{Data Analysis}

The experimenter was blind to treatment groups when taking all measures. The results are presented as mean values \pm SEM. ANOVA tests were used for multiple groups' comparison, and Bonferroni's post hoc analyses were used to assess the significance between isolated groups. Number of animals used is indicated by $n$. Probability values of $p<0.05$ were considered to represent significant differences.

\section{RESULTS}

\section{Formation of Fear Memories with Multiple Cues}

We initially determined whether our conditioning protocol with multiple environmental cues could lead to the formation of stable fear memories (Figure 1a). Electric tail shocks (US) were paired with three different conditioned cues (CS: odor, tone, and visual context) and fear memory formation was examined 1 week after conditioning training (Figure 1b-j). After the presentation of different CS separately, the plasma levels of corticosterone (an indicator of the hypothalamus-pituitary-adrenal-axis activation) were significantly increased in the conditioned rats, 


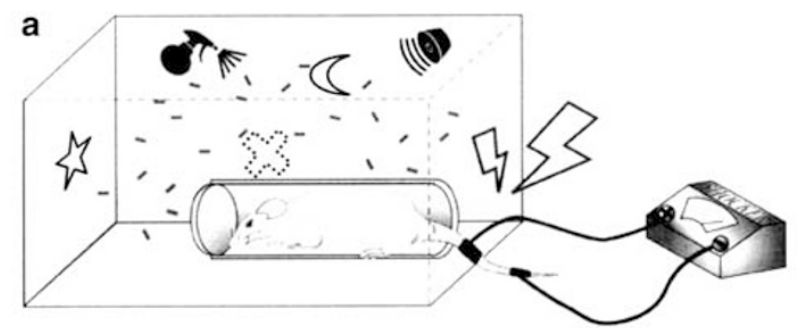

b

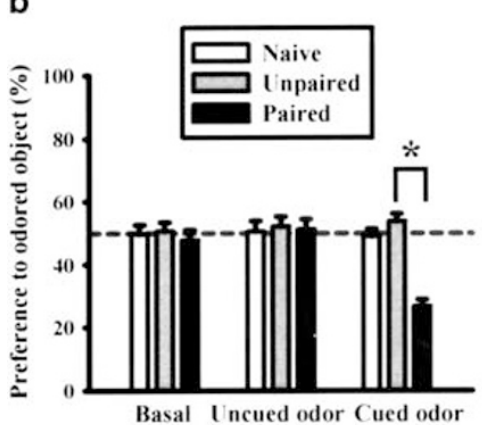

e

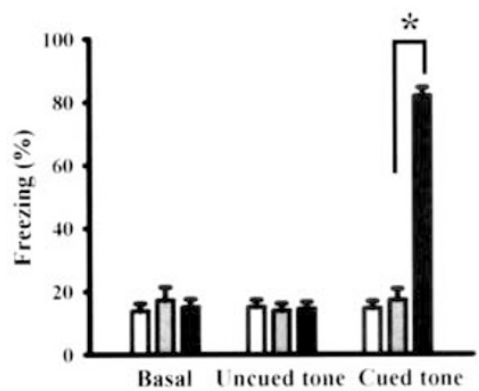

h

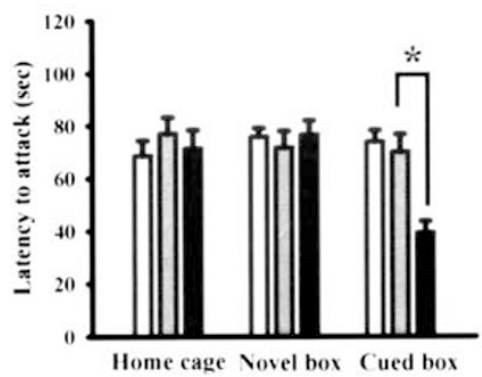

c

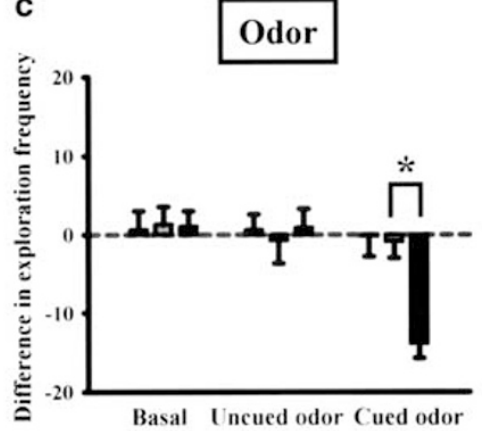

f

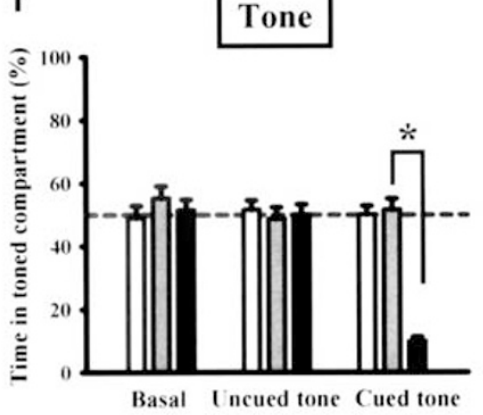

i

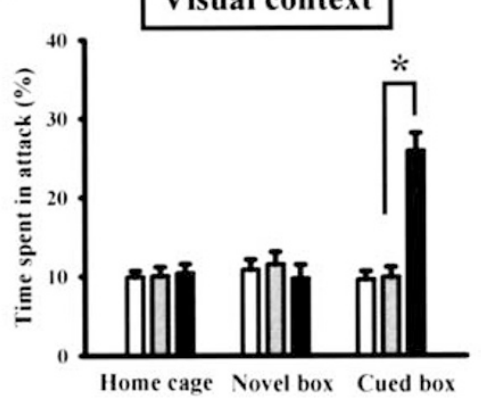

d

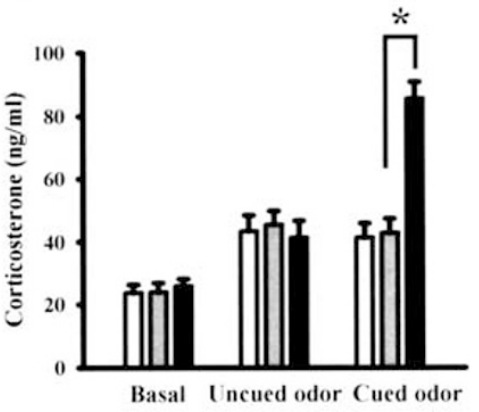

g

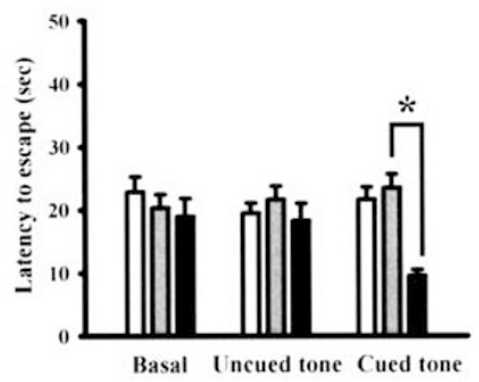

j

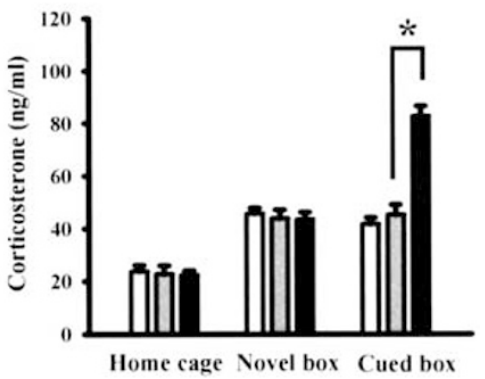

Figure I Conditioning protocol with multiple environmental cues leads to the formation of associative fear memories. (a) Rats were conditioned with three different environmental cues (CS: odor, tone, and visual context)-electric tail shock pairing (US, 60 pairings). In the experiment, rat was placed into a cylinder-shaped restrainer and the restrainer was placed on the center of a conditioning chamber. Odor cue $(0.1 \%$ Citrus bergamia) was sprayed around in the context and a tone cue $(2 \mathrm{kHz}$ at $80 \mathrm{~dB})$ was delivered conditioning just before the tail shock ( $\mathrm{mAA}, 30-90 \mathrm{~s}$ apart) through a speaker attached to the wall of the chamber. (b-d) Rats were conditioned with multiple environmental cues-electric tail shock pairing and fear memories were tested I week after fear conditioning training. The conditioned rats displayed aversive behaviors to the conditioned odor cue-paired object (reduction in relative exploration time and frequency) and an increase in the plasma corticosterone levels after re-exposure to the odor cue $(n=7$ for all experimental groups). (e-g) The conditioned rats displayed a profound increase in freezing behaviors to conditioned tone and a significant CPA to the tone cue-presented compartment ( $n=7$ for all experimental groups). ( $h-j)$ The conditioned rats showed an increase in aggressive behaviors (spent more time to attack the intruder) and an increase in the plasma corticosterone levels after re-visiting the conditioned chamber ( $n=7$ for all experimental groups). All data represent group mean values \pm SEM. $* p<0.05$ represent significant difference.

indicating that each of the CS triggered the retrieval of stressful experience (paired vs unpaired; odor: $85.4 \pm 5.2 \mathrm{vs}$ $42.7 \pm 4.6 \mathrm{ng} / \mathrm{ml}$, tone: $96.2 \pm 4.9$ vs $43.7 \pm 3.0 \mathrm{ng} / \mathrm{ml}$, visual context: $82.6 \pm 3.8$ vs $45.1 \pm 3.9 \mathrm{ng} / \mathrm{ml}, p<0.01)$. Detailed behavioral analyses were designed to study the interconnection among different CS. To evaluate the expression of conditioned responses to odor cue, an odor preference test was used (Supplementary Figure S1). We found that 
exposure to an odor cue previously paired with US resulted in strong aversion by conditioned rats to odor-labeled objects $\left(\mathrm{F}_{(2,20)}=40.5, p<0.001\right)$ and a significant decrease in exploration frequency $\left(\mathrm{F}_{(2,20)}=12.1, p<0.001\right)$. Post hoc analysis revealed a significant difference in preference to odored object (paired vs unpaired: $26.7 \pm 2.2$ vs $53.7 \pm 2.3 \%$, $p<0.01$ ) and exploration frequency (paired $v s$ unpaired: $-13.8 \pm 1.8$ vs $-0.85 \pm 2.1, p<0.01$ ) (Figure $1 b$ and $c$; Supplementary Figure S1).

Given freezing behavior is a natural representation of the emergence of fearful sensation; it has long been used as an indicator to reflect the retrieval of conditioned fear responses. To assess the ability of conditioned tone cue to retrieve fear responses, percentage of time spent in freezing behavior during the tone cue presentation period (Supplementary Figure S2) and tone-induced CPA were examined (Supplementary Figure S3). We found that exposure to the tone cue previously paired with US resulted in a profound increase in freezing behavior $\left(\mathrm{F}_{(2,20)}=167.7, p<0.001\right)$ of conditioned rats. Post hoc analysis revealed a significant difference in time spent freezing, paired $v s$ unpaired: $81.8 \pm 2.7$ vs $17.2 \pm 3.6 \%, p<0.01$. (Figure 1e; Supplementary Figure S2). Similarly, conditioned rats exhibited a significant CPA to the tone cue-presented compartment (time in toned compartment: $\mathrm{F}_{(2,20)}=73.3, p<0.001$; latency to escape: $\left.\mathrm{F}_{(2,20)}=18.2, p<0.001\right)$. Post hoc analysis revealed a significant difference in preference to toned compartment (paired vs unpaired: $9.8 \pm 1.4$ vs $51.5 \pm 3.6 \%$, $p<0.01$ ) and latency to escape (paired $v s$ unpaired: $9.5 \pm 1.0$ vs $23.5 \pm 2.1 \mathrm{~s}, p<0.01$ ) (Figure $1 \mathrm{f}$ and $\mathrm{g}$ ).

As fear and anxiety strongly affects the social and aggressive behaviors of animals (Beiderbeck et al, 2007), we used resident-intruder aggressive behavior test to examine whether the conditioned chamber may normally represent a visual context cue to retrieve conditioned fear responses. When returned to the conditioned chamber, conditioning paired rats showed significant increase in aggressive behaviors as indicated by a reduction in the latency time to the first attack $\left(\mathrm{F}_{(2,20)}=12.9, p<0.001\right)$ and more time spent in attacks on intruders $\left(\mathrm{F}_{(2,20)}=34.1\right.$, $p<0.001)$. Post hoc analysis revealed a significant difference in latency to attack (paired vs unpaired: $39.2 \pm 4.2 v s$ $69.9 \pm 6.7 \mathrm{~s}, \quad p<0.01)$ and total time spent in attack (paired vs unpaired: $25.9 \pm 2.2$ vs $9.9 \pm 1.2 \%, p<0.01$ ) (Figure $1 \mathrm{~h}$ and $\mathrm{i}$ ).

In addition, these multiple cues connected fear memories continued for $>1$ month (Supplementary Figure S4). No significant differences in the basal locomotor activity and anxiety-related behaviors in open-field test were observed in conditioned rats 1 week or 1 month after conditioning training (Supplementary Figure S5). These results suggested that our fear conditioning model by presenting several environmental stimuli during fear conditioning could effectively lead to the formation of stable fear memories.

\section{Effectiveness of Extinction Training on Removing Conditioned Fear Responses}

We next investigated whether single-cue extinction training is sufficient to extinguish conditioned fear responses (Figure 2a). The conditioned rats were repeatedly exposed to conditioned tone cue 1 week after fear conditioning training. After three extinction training sessions, both the tone cue-elicited freezing behaviors $\left(\mathrm{F}_{(2,20)}=124.3\right.$, $p<0.001)$ and motivation of avoidance to the tone-paired compartment were significantly diminished during the test sessions (time in toned compartment: $\mathrm{F}_{(2,20)}=53.3$, $p<0.001$, latency to escape: $\left.\mathrm{F}_{(2,20)}=8.8, p<0.01\right)$, reflecting the effectiveness of extinction training protocol. Post hoc analysis revealed a significant difference in time spent freezing (extinction $v s$ non-extinction: $28.1 \pm 2.7 \quad v s$ $84.9 \pm 2.8 \%, p<0.01$ ), time in toned compartment (extinction $v s$ non-extinction: $46.6 \pm 2.0$ vs $11.2 \pm 2.3 \%, p<0.01$ ) and latency to escape (extinction $v s$ non-extinction: $20.1 \pm 2.8$ vs $10.0 \pm 0.6 \mathrm{~s}, p<0.01$ ) (Figure $2 \mathrm{~b}-\mathrm{d}$ ). However, the conditioned fear responses to other cues were unchanged in rats that received extinction training (Figure 2e-j). The conditioned rats still showed an aversion toward the odor cue (extinction vs non-extinction; preference to odored object: $\mathrm{F}_{(1,13)}=0.8, p=0.39$; difference in exploration frequency: $F_{(1,13)}=0.1, p=0.9$ ) (Figure $2 \mathrm{e}-\mathrm{g}$ ) and obvious aggressive behaviors after re-exposure to the conditioned chamber (extinction $v s$ non-extinction; latency: $\mathrm{F}_{(1,13)}=0.3, p=0.6$; time spent in attack: $\mathrm{F}_{(1,13)}=1.5$, $p=0.25$ ) (Figure $2 \mathrm{~h}$ and i). Similar cue-specific extinction on conditioned fear responses was also observed after repeatedly training with a conditioned odor cue (Supplementary Figure S6).

As adjunctive DCS has been reported to effectively strengthen extinction of conditioned fear responses (Walker et al, 2002; Ledgerwood et al, 2005), we next determined whether the combination of DCS treatment with single-cue extinction training may lead to a generalized suppression of conditioned responses to all paired conditioned cues. For this purpose, conditioned rats were injected intraperitoneally with DCS $(30 \mathrm{mg} / \mathrm{kg}) 30 \mathrm{~min}$ before the start of extinction training with conditioned tone cue. We found that the level of tone cue-induced freezing behaviors and avoidance behaviors were significantly lower in the extinction training groups than in the non-extinction training groups (Supplementary Figure S7b-d). Unfortunately, the conditioned responses elicited by other cues remained unchanged after extinction training. The conditioned rats still showed aversion to the odor cue (extinction-DCS $v s$ non-extinction-DCS; preference to odored object: $\mathrm{F}_{(1,13)}=1.7, p=0.22$; difference in exploration frequency: $\mathrm{F}_{(1,13)}=0.1, p=0.97$ ) (Supplementary Figure S7e-g) and aggressive behaviors after re-exposure to the conditioned chamber (extinction-DCS $v s$ non-extinction-DCS; latency to attack: $\mathrm{F}_{(1,13)}=0.2, \quad p=0.67$; time spent in attack: $\mathrm{F}_{(1,13)}=0.2, p=0.63$ ) (Supplementary Figure $\mathrm{S} 7 \mathrm{~h}-\mathrm{j}$ ).

\section{Effectiveness of the Disruption of Reconsolidation Process on Removing Conditioned Fear Responses}

We then determined whether the disruption of memory reconsolidation process may lead to generalized inhibition of conditioned fear responses. Since the memory reconsolidation requires de novo protein synthesis (Nader et al, 2000a), we used protein synthesis inhibitors to interfere with the reconsolidation process. As the BLA is a major emotional center in the brain and has an essential role in memory formation and storage of fear conditioning (Fanselow and LeDoux, 1999), we examined whether the 

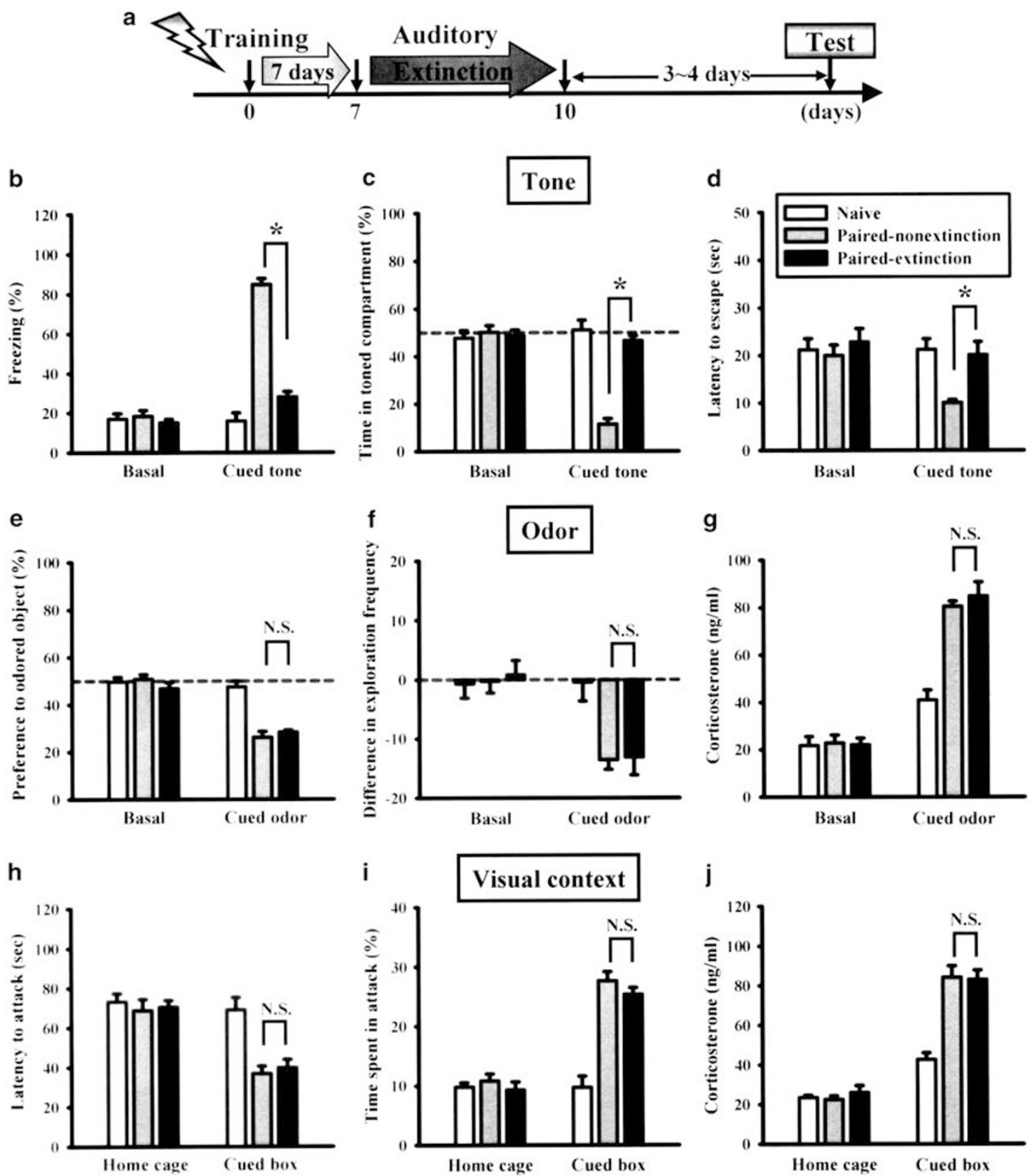

Figure 2 Single-cue extinction training reduced the expression of the conditioned fear responses in a cue-specific manner. (a) Rats were conditioned with three different environmental cues-electric tail shock pairing. One week later, they were received extinction trials with repetitive tone cue re-exposure. Memory retention was tested 3 days later. $(b-d)$ The conditioned rats with extinction training showed a significant reduction in both freezing behaviors and motivation of avoidance to tone cue-paired compartment ( $n=7$ for all experimental groups). (e-g) The conditioned rats with extinction training displayed aversive behaviors to the conditioned odor cue-paired object and a robust increase in the plasma corticosterone levels after re-exposure to the odor cue ( $n=7$ for all experimental groups). ( $h-j)$ The conditioned rats with extinction training displayed an increase in aggressive behaviors and the plasma corticosterone levels after re-visiting the conditioned chamber $(n=7$ for all experimental groups). All data represent group mean values \pm SEM. $* p<0.05$. N.S., not significant.

BLA is also important in the reconsolidation of fear memories in our model. Initially, multiple conditioning cues were paired with tail shocks to form fear memories (Figure 3a). One week after fear conditioning training, a protein synthesis inhibitor, cycloheximide $(5 \mu \mathrm{g})$ was bilaterally injected into the BLA immediately after tone cue-induced memory recall and memory retention was tested 3-4 days later. We found that disrupting memory reconsolidation process by means of cycloheximide effectively attenuated tone cue-induced conditioned responses (freezing: $\mathrm{F}_{(3,27)}=69.4, p<0.001$, time in toned compartment: $\mathrm{F}_{(3,27)}=28.5, p<0.001$; latency to escape: $\mathrm{F}_{(3,27)}=6.9$, $p<0.01)$. Post hoc analysis revealed a significant difference in time spent freezing (paired-cycloheximide $v s$ pairedvehicle: $35.7 \pm 2.9$ vs $76.0 \pm 3.6 \%, p<0.01)$, time in toned compartment (paired-cycloheximide $v s$ paired-vehicle: 
a
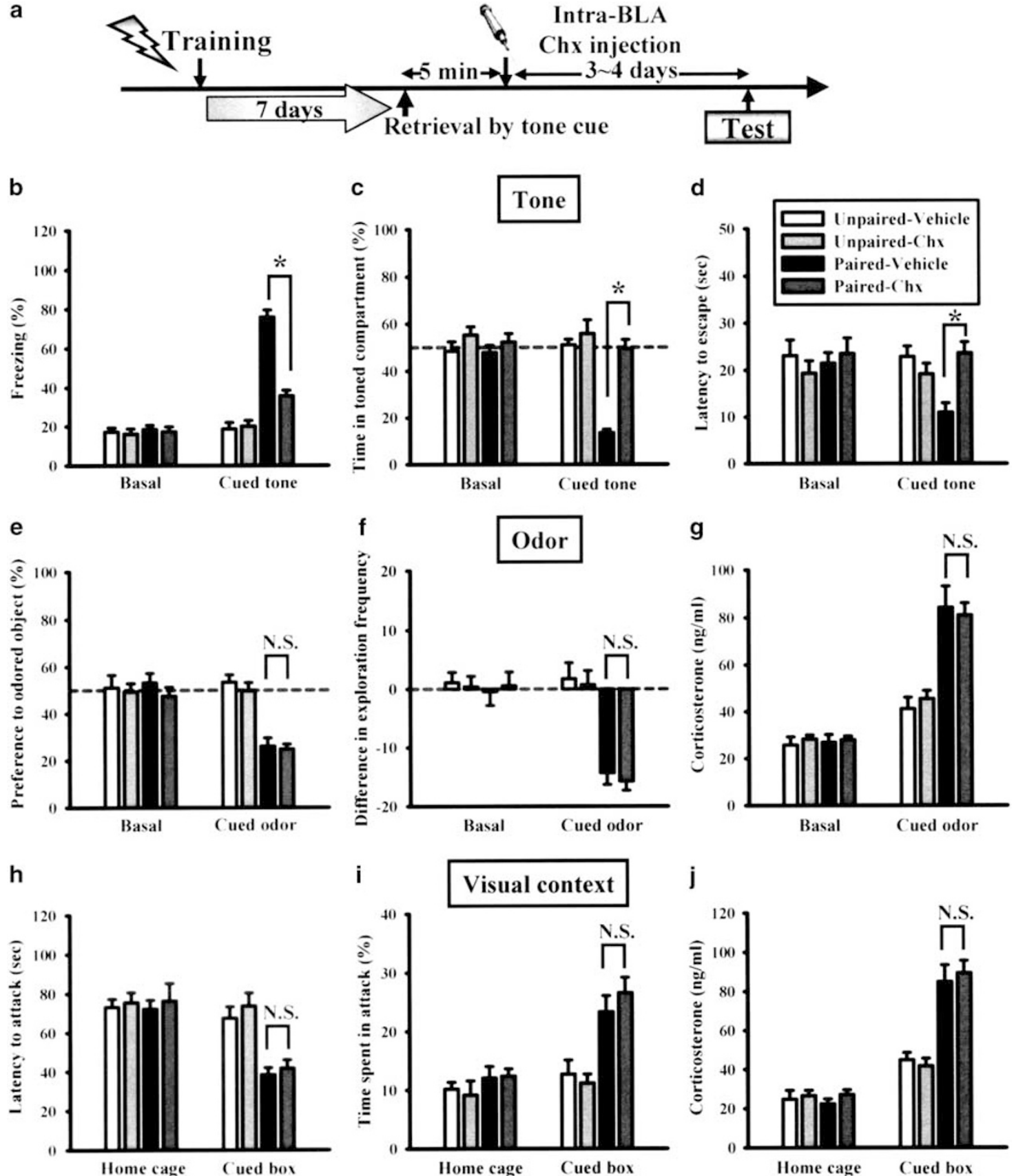

Figure 3 Intra-BLA injection of cycloheximide during memory recall disrupts memory reconsolidation specific to the reactivated memory trace. (a) Experimental designs for fear memory conditioning and reconsolidation targeting in the BLA. One week after fear conditioning with multiple cues, fear memories were recalled by a brief tone cue re-exposure $(90 \mathrm{~s})$ and rats were then intra-BLA injected with cycloheximide (Chx, $5 \mu \mathrm{g})$ to disrupt memory reconsolidation process. Memory retention was tested 3 days later. $(b-d)$ The conditioned rats with BLA Chx injected showed a significant reduction in both freezing behaviors and escaping behaviors to the tone cue-paired compartment compared with the vehicle injection groups ( $n=7$ for all experimental groups). (e-g) The conditioned rats with $\mathrm{Chx}$ injection showed aversive behaviors to the conditioned odor cue-paired object and an increase in the plasma corticosterone levels after re-exposure to the odor cue ( $n=7$ for all experimental groups). (h-j) The conditioned rats with Chx injection displays high levels of aggressive behaviors and an increase in the plasma corticosterone levels after re-visiting the conditioned chamber ( $n=7$ for all experimental groups). All data represent group mean values \pm SEM. ${ }^{*} p<0.05$. N.S., not significant.

$49.5 \pm 3.8$ vs $13.5 \pm 1.4 \%, p<0.01)$ and latency to escape (paired-cycloheximide vs paired-vehicle: $23.6 \pm 2.4$ vs $10.9 \pm 2.0 \mathrm{~s}, p<0.01$ ) (Figure $3 \mathrm{~b}-\mathrm{d}$ ). To determine whether disrupting BLA-protein synthesis-dependent reconsolidation process could generalize the suppression of conditioned fear responses to other cues, the responses to the other two reminder cues were tested. Unfortunately, the conditioned fear responses to other cues remained unchanged. After re-exposure to the conditioned odor cue, the conditioned rats that received bilateral injections of cycloheximide into the BLA still showed an aversion toward the odor cue (paired-cycloheximide vs paired-vehicle; 
preference to odored object: $\mathrm{F}_{(1,13)}=0.1, p=0.77$; difference in exploration frequency: $\mathrm{F}_{(1,13)}=0.3, p=0.59$ ) (Figure $3 \mathrm{e}-\mathrm{g}$ ) and aggressive behaviors after re-exposure to the conditioned chamber (paired-cycloheximide vs pairedvehicle; latency to attack: $\mathrm{F}_{(1,13)}=0.3, p=0.57$, time spent in attack: $\left.F_{(1,13)}=0.7, p=0.41\right)$ (Figure $3 \mathrm{~h}-\mathrm{j}$ ).

In addition to the BLA, the $\mathrm{DH}$ has been implicated in memory reconsolidation processes (Kida et al, 2002; Debiec et al, 2002; Kelly et al, 2003). Therefore, we investigated whether pharmacological intervention of $\mathrm{DH}$-dependent protein synthesis-dependent reconsolidation process may result in generalization of fear inhibition. To this end, 1 week after fear conditioning training, cycloheximide was bilaterally injected into the CA1 region of the $\mathrm{DH}$ immediately after tone cue-induced memory recall and memory retention was tested 3 days later. We found that disrupting DH-dependent memory reconsolidation process successfully attenuated tone cue-induced conditioned responses (freezing: $\mathrm{F}_{(3,27)}=116.4, p<0.001$; time in toned compartment: $\mathrm{F}_{(3,27)}=49.1, p<0.001$; latency to escape: $\left.\mathrm{F}_{(3,27)}=5.9, p<0.01\right)$. Post hoc analysis revealed a significant difference in time spent freezing (paired-cycloheximide $v s$ paired-vehicle: $30.5 \pm 2.4$ vs $82.1 \pm 3.4 \%, p<0.01$ ); time in toned compartment (paired-cycloheximide $v s$ paired-vehicle: $54.8 \pm 3.3$ vs $12.7 \pm 2.1 \%, p<0.01$ ) and latency to escape (paired-cycloheximide $v s$ paired-vehicle: $18.6 \pm 2.3 v s$ $9.2 \pm 1.1 \mathrm{~s}, p<0.01$ ) (Figure $4 \mathrm{~b}-\mathrm{d}$ ). Correspondingly, injection of cycloheximide also inhibited the de novo synthesis of c-fos protein in the $\mathrm{DH}$ induced by memory recall (Supplementary Figure S8). To determine whether disrupting $\mathrm{DH}$-dependent protein synthesis-dependent reconsolidation process may reduce the conditioned responses to other paired cues, the conditioned responses to other cues were tested. In contrast to the cue-specific effect of disrupting protein synthesis in the BLA, intra-DH injection of cycloheximide resulted in generalized suppression of all learned associations between conditioned cues and fear memories. The fear responses to odor and visual context cues were significantly reduced. The conditioned rats that received intra-DH injections of cycloheximide showed less aversion toward the odor cue (preference to odored object: $\mathrm{F}_{(3,27)}=61.6, p<0.001$; difference in exploration frequency: $\left.\mathrm{F}_{(3,27)}=11.1, p<0.001\right)$. Post hoc analysis revealed a significant difference in preference to odored object (paired-cycloheximide vs paired-vehicle: $53.5 \pm 1.9 v s$ $23.0 \pm 1.3 \%, p<0.01$ ) and exploration frequency (pairedcycloheximide $v s$ paired-vehicle: $0.86 \pm 2.3 v s-13.6 \pm 1.5$, $p<0.01$ ) (Figure $4 \mathrm{e}-\mathrm{g}$ ) and less aggressive behaviors after re-exposure to the conditioned chamber (latency to attack: $\mathrm{F}_{(3,27)}=13.8, p<0.001$; time spent in attack: $\mathrm{F}_{(3,27)}=13.6$, $p<0.001)$. Post hoc analysis revealed a significant difference in latency to attack (paired-cycloheximide $v s$ paired-vehicle: $73.3 \pm 5.0$ vs $37.1 \pm 4.3 \mathrm{~s}, p<0.01)$ and time spent in attack (paired-cycloheximide $v s$ paired-vehicle: $10.2 \pm 1.9$ vs $24.3 \pm 2.4 \%, p<0.01$ ) (Figure $4 \mathrm{~h}-\mathrm{j}$ ). Furthermore, the effect of reconsolidation blocking treatment displayed a universal feature and could be recapitulated by using other conditioned cues for memory recall (Supplementary Figure S9) or protein synthesis inhibitor anisomycin (Supplementary Figure S10). The disrupting effect of cycloheximide on fear memory expression was not due to its non-specific effects, because cycloheximide injection without memory trace recall had no significant effect on fear memory retention (Supplementary Figure S11).

CREB-mediated gene expression has long been implicated in fear memory consolidation (Korzus et al, 2004; Mamiya et al, 2009). To determine whether transcriptional activation by CREB is also required for fear memory reconsolidation, we examined the effect of genetic suppression of CREB function on previously consolidated fear memories. A lentiviral gene delivery system was used for regional and temporal in vivo transgenic targeting. Two days after fear conditioning training, lentiviruses stably expressing the KCREB were bilaterally injected into the CA1 region of the DH (Figure 5a). One week later, the conditioned rats were re-exposed to the conditioned tone cue and memory retention was examined 3 days later (Figure 5b). Memory retention test results revealed that expression of KCREB in the DH effectively disrupted tone cue-induced fear responses (freezing: $\mathrm{F}_{(2,20)}=86.4, p<0.001$; time in toned compartment: $\mathrm{F}_{(2,20)}=70.5, p<0.001$; latency to escape: $\left.\mathrm{F}_{(2,20)}=10.8, \quad p<0.001\right)$. Post hoc analysis revealed a significant difference in time spent freezing (paired-KCREB vs paired-EGFP: $33.2 \pm 2.1$ vs $78.7 \pm 3.5 \%, p<0.01$ ); time in toned compartment (paired-KCREB vs paired-EGFP: $47.2 \pm 3.2 v s 12.8 \pm 1.8 \%, p<0.01)$ and latency to escape (paired-KCREB vs paired-EGFP: $20.5 \pm 2.4$ vs $9.2 \pm 2.1 \mathrm{~s}$, $p<0.01$ ) (Figure 5c-e). Correspondingly, overexpressing KCREB also inhibited the de novo synthesis of c-fos protein in the DH induced by memory recall (Supplementary Figure S12a and b). Like cycloheximide did, disrupting memory reconsolidation process by decreasing CREB function led to a generalized loss of all learned associations between conditioned cues and the established fear memories. The conditioned rats that received intra-DH injection of KCREB showed less aversion toward the odor cue (preference to odored object: $\mathrm{F}_{(2,20)}=26, p<0.001$; difference in exploration frequency: $\left.\mathrm{F}_{(2,20)}=7.1, p<0.01\right)$. Post hoc analysis revealed a significant difference in preference to odored object (paired-KCREB vs paired-EGFP: $52.8 \pm 4.3 \%$ vs $26.1 \pm 1.7 \%, p<0.01$ ) and exploration frequency (pairedKCREB $v s$ paired-EGFP: $-1.3 \pm 1.6 v s-13.6 \pm 3.1, p<0.01$ ) (Figure $5 \mathrm{f}-\mathrm{h}$ ) and less aggressive behaviors after reexposure to the conditioned chamber (latency to attack: $\mathrm{F}_{(2,20)}=4.9, p<0.05$; time spent in attack: $\mathrm{F}_{(2,20)}=14.7$, $p<0.001)$. Post hoc analysis revealed a significant difference in latency to attack (paired-KCREB vs paired-EGFP: $67.7 \pm 9.7 v s 40.4 \pm 3.8 \mathrm{~s}$ ) and time spent in attack (pairedKCREB vs paired-EGFP: $11.1 \pm 1.8$ vs $24.3 \pm 2.6 \%, p<0.01$ ) (Figure 5i-k).

To further address the role of the BLA in the reconsolidation process of fear memories, we further examined the effect of genetic suppression of CREB function in the BLA on previously consolidated fear memories. Two days after fear conditioning training, lentiviruses stably expressing KCREB were bilaterally injected into the BLA (Figure 6a). One week later, the conditioned rats were re-exposed to the conditioned tone cue and memory retention was examined 3 days later (Figure 6b). We found that expression of KCREB in the BLA effectively disrupted conditioned tone cue-induced fear responses (freezing: $F_{(2,20)}=46.4$, $p<0.001$, time in toned compartment: $F_{(2,20)}=65.5$, $p<0.001$; latency to escape: $\left.\mathrm{F}_{(2,20)}=11.3, p<0.001\right)$. Post hoc analysis revealed a significant difference in time spent 
a

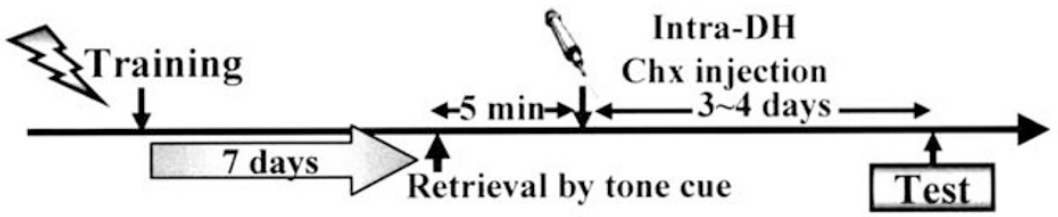

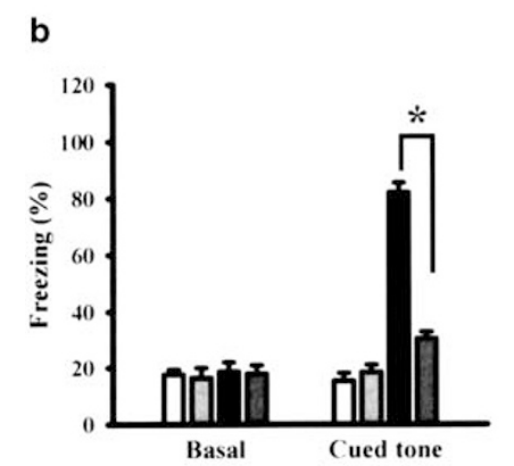
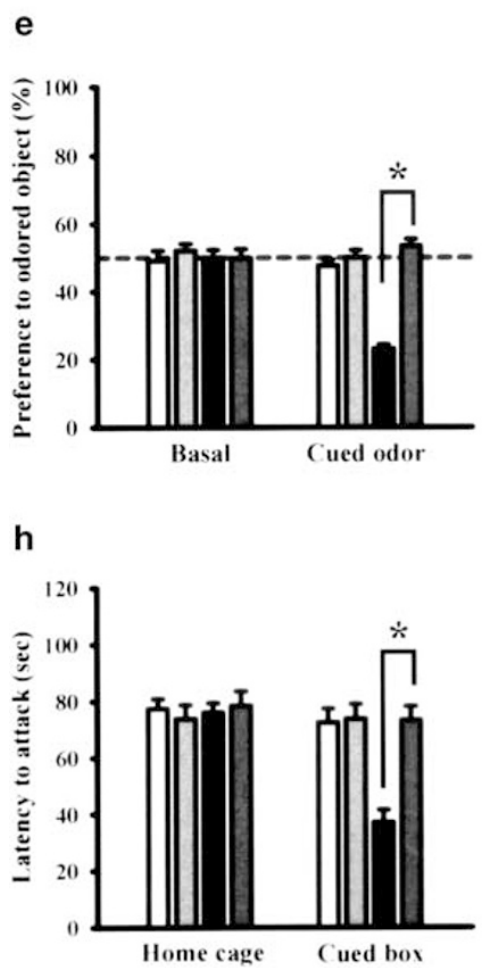
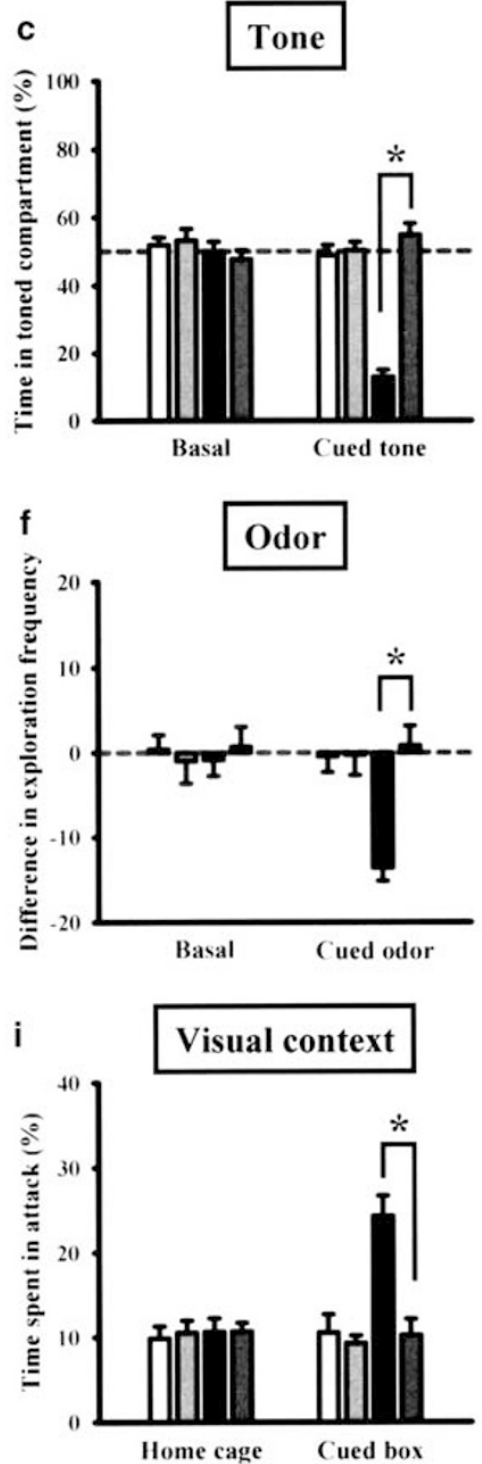

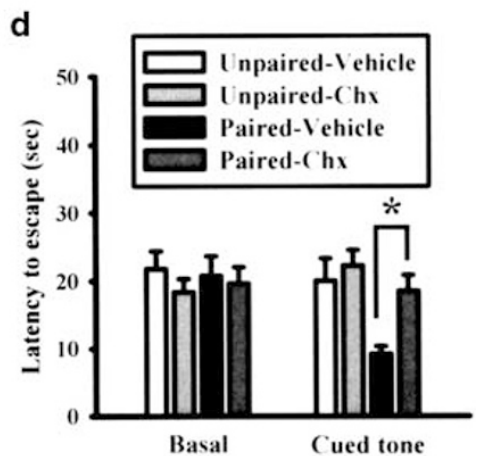

g
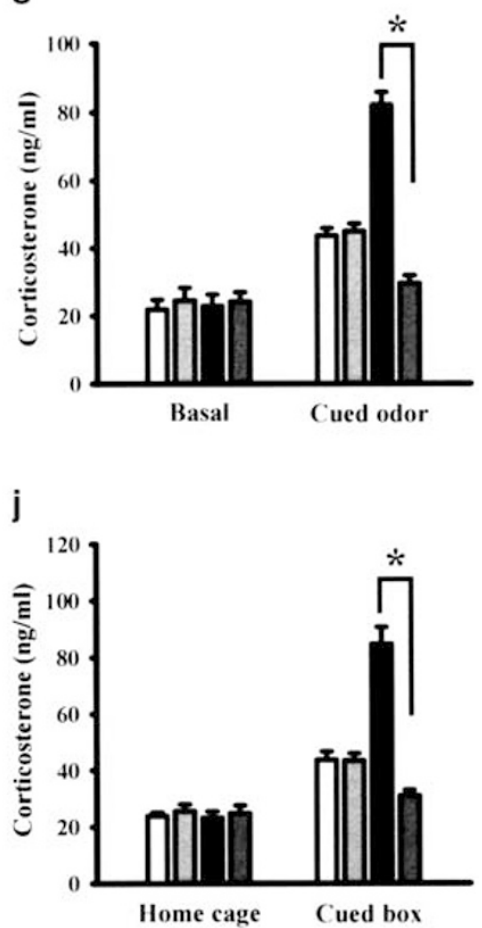

Figure 4 Intra-DH injection of cycloheximide during memory recall results in a generalized inhibition of fear responses. (a) Experimental designs for fear memory conditioning and reconsolidation targeting in the $\mathrm{DH}$. One week after fear conditioning, fear memories were recalled by a brief tone cue reexposure ( $90 \mathrm{~s}$ ) and rats were then intra-DH injected with Chx to disrupt memory reconsolidation process. Memory retention was tested 3 days later. (b-d) The conditioned rats with $\mathrm{Chx}$ injection exhibited a significant reduction in both freezing behaviors and avoidance motivation to tone cue-paired compartment compared with the vehicle injection groups ( $n=7$ for all experimental groups). (e-g) The conditioned rats with Chx injection showed less aversive behaviors to the conditioned odor cue-paired objects and a less obvious rise in the plasma corticosterone levels after re-exposure to the odor cue ( $n=7$ for all experimental groups). ( $h-j)$ The conditioned rats with Chx injection showed reduction in their aggressive behaviors and a less obvious rise in the plasma corticosterone levels after re-visiting the conditioned chamber ( $n=7$ for all experimental groups). All data represent group mean values \pm SEM. *p $<0.05$.

freezing (paired-KCREB vs paired-EGFP: $32.1 \pm 2.2 \quad v s$ $74.2 \pm 3.6 \%, p<0.01$ ), time in toned compartment (pairedKCREB $v s$ paired-EGFP: $51.9 \pm 3.6$ vs $14.3 \pm 1.4 \%, p<0.01$ ) and latency to escape (paired-KCREB vs paired-EGFP: $19.8 \pm 1.6$ vs $11.2 \pm 1.8 \mathrm{~s}, p<0.01$ ) (Figure $6 \mathrm{c}-\mathrm{e}$ ). However, the responses to other cues remained unchanged. After exposure to the odor cue, the conditioned rats that received bilateral injections of KCREB into the BLA still showed an aversion toward the odor cue (paired-KCREB vs pairedEGFP; preference to odored object: $\mathrm{F}_{(1,13)}=0.1, p=0.73$, difference in exploration frequency: $\left.\mathrm{F}_{(1,13)}=0.2, p=0.67\right)$ (Figure 6f-h) and aggressive behaviors after re-exposure to 
a

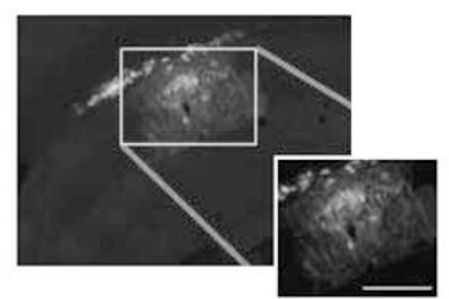

C

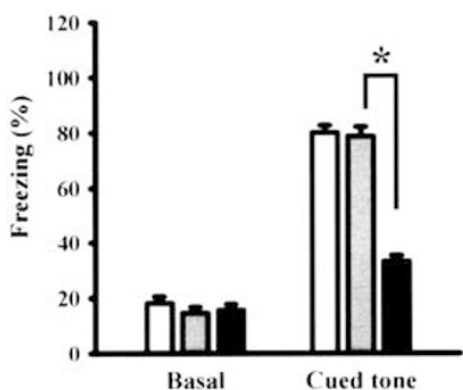

f

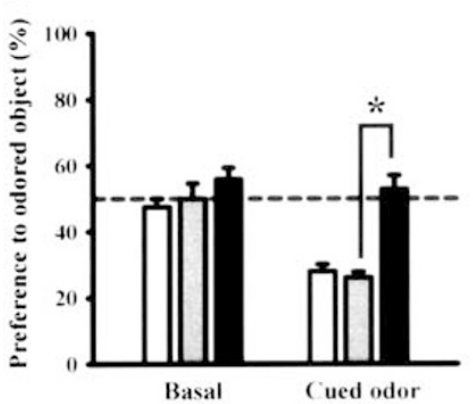

i

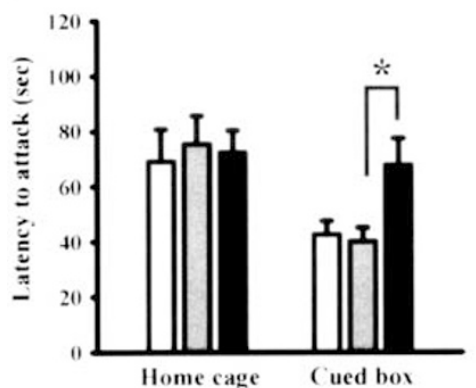

b

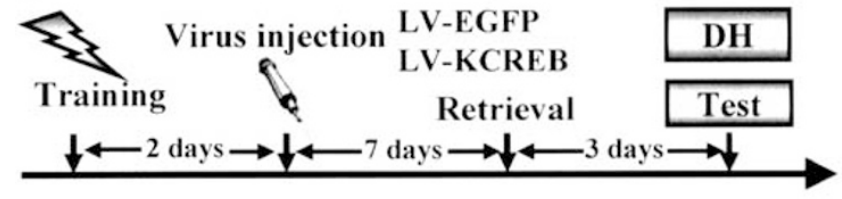

d

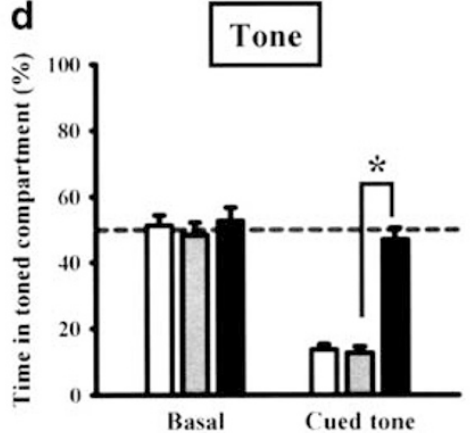

g

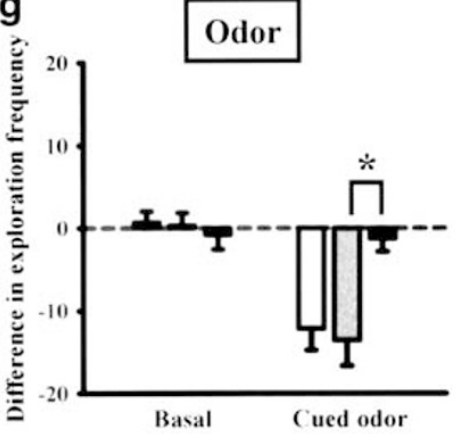

j

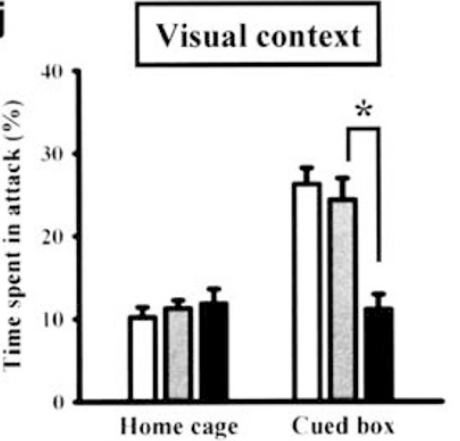

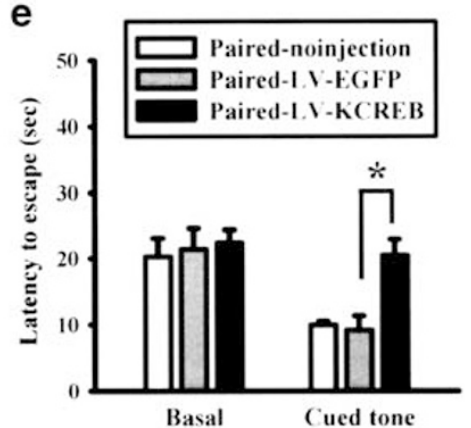

h

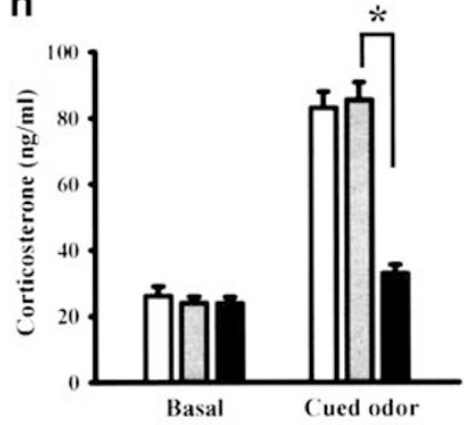

k

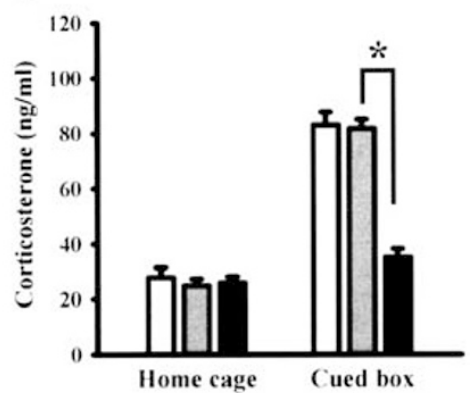

Figure 5 Intra-DH overexpression of KCREB during memory recall disrupts memory reconsolidation process and elicits a generalized inhibition of fear responses. (a) Representative images showing the delivery of transgenes (EGFP-conjugated) targeting to DH CAI region by lentivirus. Scale bar, $500 \mu$ m. (b) Experimental designs for fear memory conditioning and reconsolidation targeting. Forty-eight hours after fear conditioning, lentiviruses overexpressing KCREB (LV-KCREB) were injected locally into the DH. One week after virus injection, fear memories were recalled by a brief tone cue re-exposure ( 90 s). Memory retention was tested 3 days later. (c-e) The conditioned rats with LV-KCREB injection showed a significant reduction in both freezing behaviors and motivation of avoidance to tone cue-paired compartment compared with the LV-EGFP injection groups ( $n=7$ for all experimental groups). (f-h) The conditioned rats with LV-KCREB injection showed less aversive behaviors to the conditioned odor cue-paired object and a less obvious rise in the plasma corticosterone levels after re-exposure to the odor cue compared with the LV-EGFP-injected groups $(n=7$ for all experimental groups). (i-k) The conditioned rats with LV-KCREB injection showed a reduced amount of aggressive behaviors and a less obvious rise in the plasma corticosterone levels after re-visiting the conditioned chamber compared with the LV-EGFP-injected groups ( $n=7$ for all experimental groups). All data represent group mean values \pm SEM. $* p<0.05$

the conditioned chamber (paired-KCREB $v s$ paired-EGFP; latency to attack: $\mathrm{F}_{(1,13)}=0.6, p=0.47$, time spent in attack: $\mathrm{F}_{(1,13)}=1.0, p=0.34$ ) (Figure 6i-k). Since rats with KCREB overexpression in the DH or BLA were able to learn contextual fear conditioning following retraining, the impaired expression of cue-induced fear responses was not attributable to permanent damage to the DH or BLA (Supplementary Figure S12c and d). 


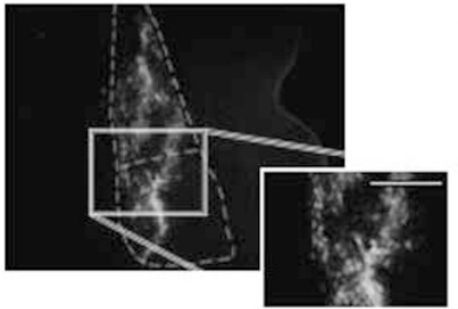

c

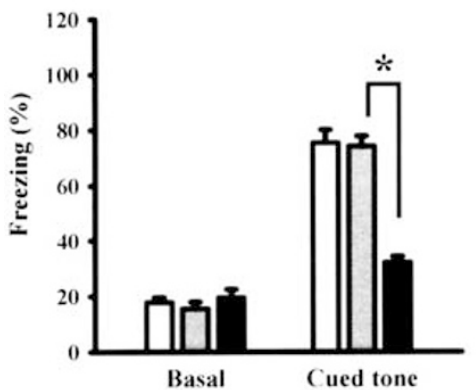

f

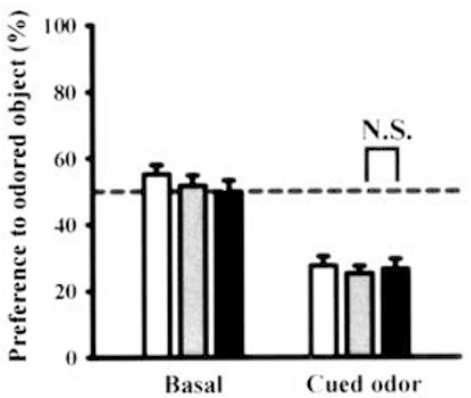

i

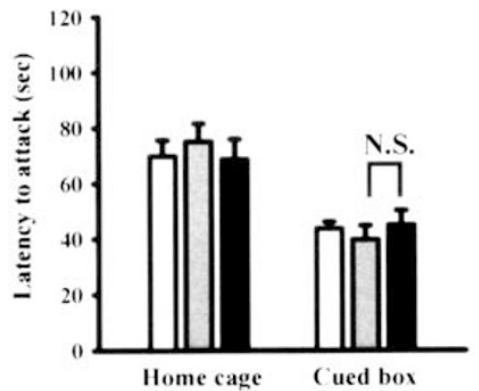

b

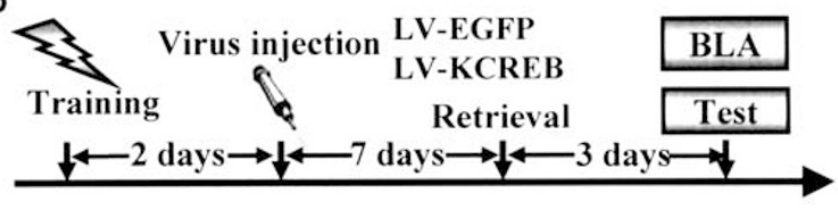

\section{d}
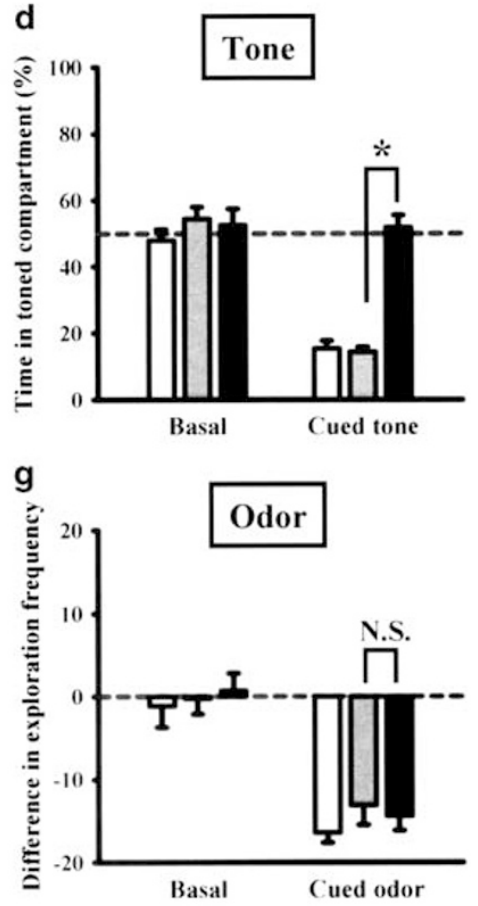

j

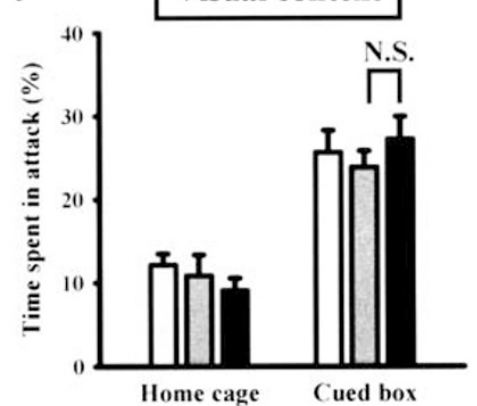

e

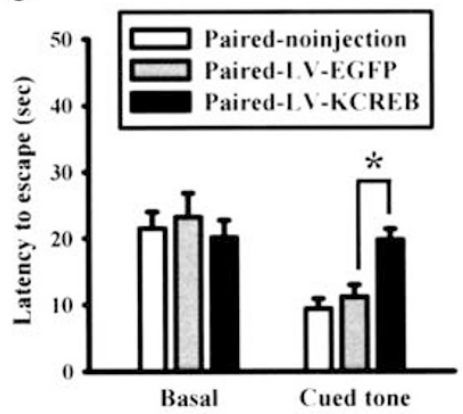

h

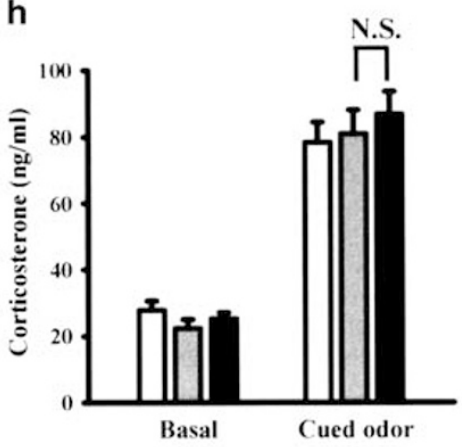

k

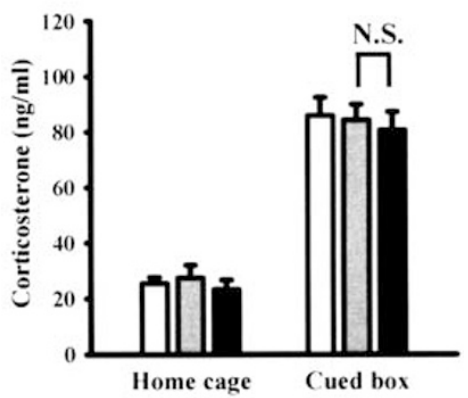

Figure 6 Intra-BLA overexpression of KCREB during memory recall disrupts memory reconsolidation in a reactivated cue-specific manner. (a) Representative images showing the delivery of transgenes (EGFP-conjugated) targeting to the BLA by lentivirus. Scale bar, $500 \mu \mathrm{m}$. (b) Experimental designs for fear memory conditioning and reconsolidation targeting in the BLA. Forty-eight hours after multiple cues fear conditioning, LV-KCREB was injected locally into the BLA. One week after virus injection, fear memories were recalled by a brief tone cue re-exposure ( $90 \mathrm{~s})$. Memory retention was tested 3 days later. (c-e) The conditioned rats with LV-KCREB injection in the BLA showed a remarkable reduction in their freezing behaviors and escaping behaviors to tone cue-paired compartment compared with the LV-EGFP injection groups ( $n=7$ for all experimental groups). ( $f-h)$ The conditioned rats with LV-KCREB injection in the BLA displayed aversive behaviors to the conditioned odor cue-paired object and an increase in the plasma corticosterone levels after reexposure to the odor cue ( $n=7$ for all experimental groups). (i-k) The conditioned rats with LV-KCREB injection in the BLA displayed an increase in aggressive behaviors and an increase in the plasma corticosterone levels after re-visiting the conditioned chamber ( $n=7$ for all experimental groups). All data represent group mean values \pm SEM. $*$ p $<0.05$. N.S., not significant.

\section{Disrupting Reconsolidation Process in the DH Prevents the Return of Fear}

An inherent problem with the inhibition of the conditioned fear responses is that the fear may return due to spontaneous recovery, reinstatement, or renewal (Effting and Kindt, 2007; Schiller et al, 2008). We next proceeded to determine whether our pharmacological intervention of $\mathrm{DH}$ protein synthesis-dependent reconsolidation process may persistently prevent the return of fear. To assess the 
recovery of fear, one additional group of rats were trained according to our previous fear conditioning paradigm (Figure 7a). One week after fear conditioning training, cycloheximide $(5 \mu \mathrm{g})$ was bilaterally injected into the CA1 region of the $\mathrm{DH}$ immediately after tone cue-induced memory recall and memory retention was tested 1 month later. We found that the conditioned rats that received bilateral injections of cycloheximide into the $\mathrm{DH}$ showed no tone cue-induced conditioned responses (freezing:
$\mathrm{F}_{(3,27)}=198.7, \quad p<0.001 ;$ time in toned compartment: $\mathrm{F}_{(3,27)}=19.5, p<0.001$; latency to escape: $\mathrm{F}_{(3,27)}=9.1$, $p<0.001)$. Post hoc analysis revealed a significant difference in time spent freezing (paired-cycloheximide $v s$ pairedvehicle: $32.8 \pm 1.9$ vs $80.7 \pm 2.0 \%, p<0.01)$, time in toned compartment (paired-cycloheximide $v s$ paired-vehicle: $49.4 \pm 3.9 v s 15.4 \pm 2.7 \%, p<0.01)$ and latency to escape (paired-cycloheximide $v s$ paired-vehicle: $21.9 \pm 2.1 \quad v s$ $10.4 \pm 1.7 \mathrm{~s}, \quad p<0.01$ ) (Figure $7 \mathrm{~b}-\mathrm{d}$ ). In addition, the
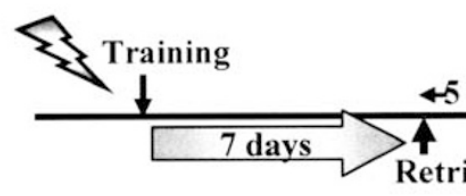
$-5 \mathrm{~min} \rightarrow \mathrm{d}$ Retrieval by tone cue

b

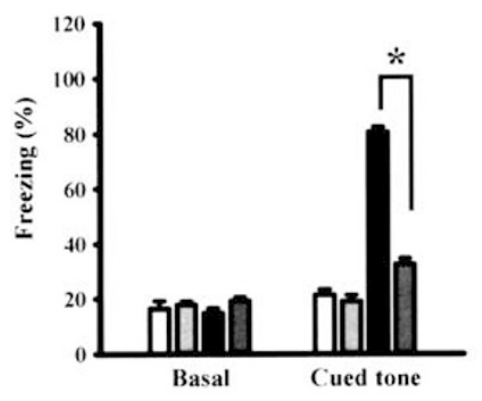

e

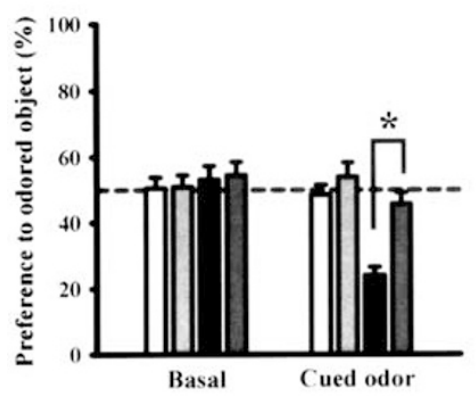

h

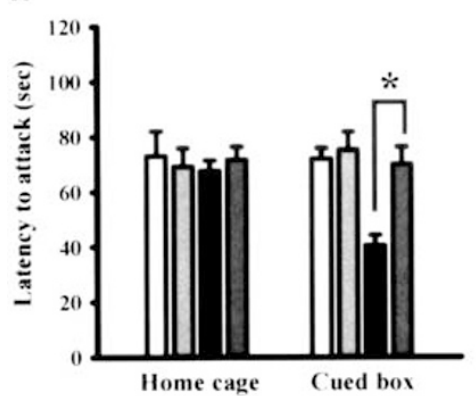

C

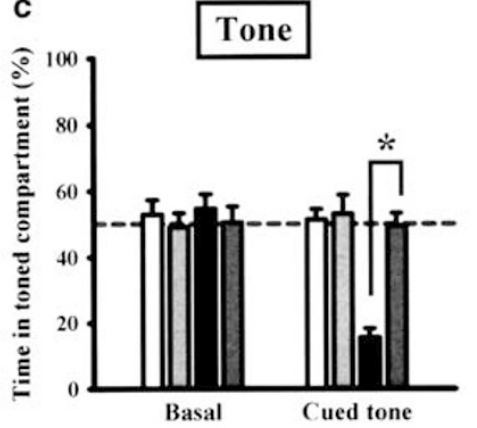

f

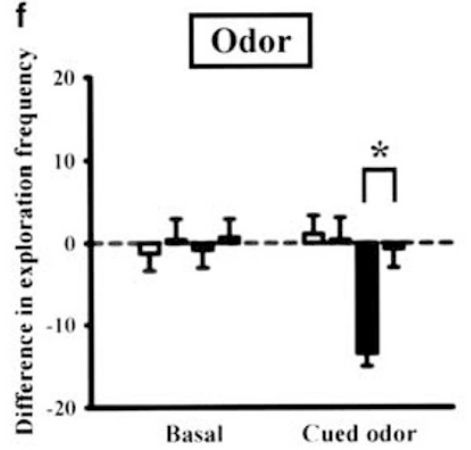

i

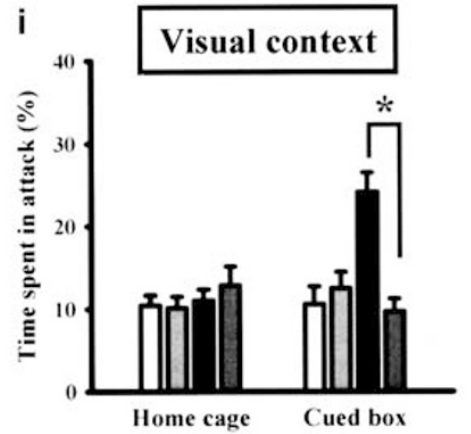

Intra-DH

Chx injection

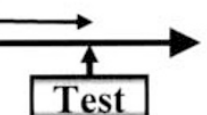

d

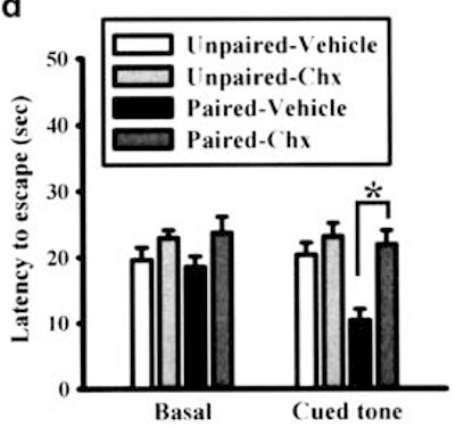

g

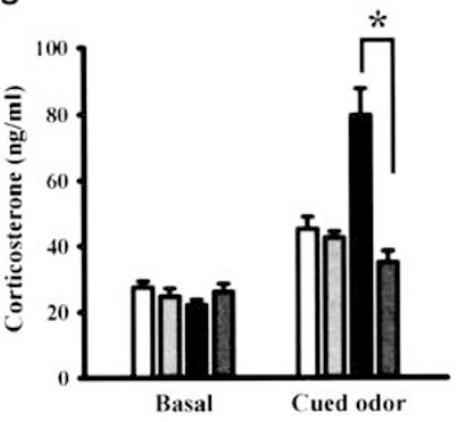

j

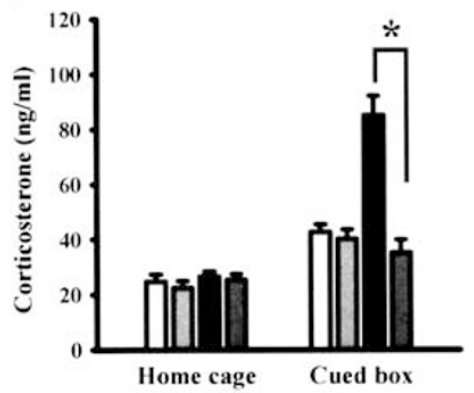

Figure 7 Suppression of the spontaneous recovery of fear responses by blocking the reconsolidation process in the DH. (a) Experimental designs for fear memory conditioning and testing for the recovery of suppressed fear memories. One week after fear conditioning training, fear memories were recalled by a brief tone cue re-exposure $(90 \mathrm{~s})$ and rats were then intra-DH injected with $\mathrm{Chx}$ to disrupt memory reconsolidation process. The recovery of suppressed fear memories ware tested I month later. (b-d) The conditioned rats with Chx injection displayed a significant suppression of freezing behaviors and avoidance motivation to tone cue-paired compartment compared with the vehicle-injected groups ( $n=7$ for all experimental groups). (e-g) The conditioned rats with $\mathrm{Chx}$ injection showed less aversive behaviors to the conditioned odor cue-paired objects and a less obvious rise in the plasma corticosterone levels after re-exposure to the odor cue ( $n=7$ for all experimental groups). ( $h-j)$ The conditioned rats with Chx injection displayed a reduced amount of aggressive behaviors and a less obvious rise in the plasma corticosterone levels after re-visiting the conditioned chamber $(n=7$ for all experimental groups). All data represent group mean values \pm SEM. $* p<0.05$. 
conditioned fear responses to the other cues remained suppressed in cycloheximide-treated conditioned rats. The conditioned rats that received intra-DH injections of cycloheximide showed less aversion toward the odor cue (preference to odored object: $\mathrm{F}_{(3,27)}=15.9, p<0.001$; difference in exploration frequency: $F_{(3,27)}=9.8$, $p<0.001)$. Post hoc analysis revealed a significant difference in preference to odored object (paired-cycloheximide $v s$ paired-vehicle: $45.7 \pm 3.4$ vs $23.9 \pm 2.5 \%, p<0.01)$ and exploration frequency (paired-cycloheximide $v s$ pairedvehicle: $-0.7 \pm 2.3 v s-13.4 \pm 1.6, p<0.01$ ) (Figure $7 \mathrm{e}-\mathrm{g}$ ) and less aggressive behaviors after re-exposure to the conditioned chamber (latency to attack: $\mathrm{F}_{(3,27)}=9.3$, $p<0.001$; time spent in attack: $\left.\mathrm{F}_{(3,27)}=11.3, p<0.001\right)$. Post hoc analysis revealed a significant difference in latency to attack (paired-cycloheximide $v s$ paired-vehicle: $70.1 \pm 6.2$ vs $40.4 \pm 3.8 \mathrm{~s}, p<0.01$ ) and time spent in attack (pairedcycloheximide vs paired-vehicle: $9.7 \pm 1.6$ vs $24.2 \pm 2.3 \%$, $p<0.01$ ) (Figure $7 \mathrm{~h}-\mathrm{j}$ ).

\section{DISCUSSION}

Repetitive replay of past stressful experiences may affect the mental health status and contribute to the etiology of PTSD and other anxiety disorders. There has been considerable interest in the use of extinction training or disrupting memory reconsolidation process to prevent the re-emergence of fear memories. However, growing evidence from both animal and human studies has revealed that extinction training has limited ability to eliminate fear memories. In this study, we show that combining extinction training with DCS reduces cue-specific conditioned responses but fails to erase fear memories. By contrast, temporally and regionally restricted disruption of $\mathrm{DH}$-dependent reconsolidation process by means of protein synthesis inhibitors or genetic suppression of CREB function comprehensively diminished the learned connections between fear responses and all paired conditioned cues. We therefore hypothesize that disrupting reconsolidation process is preferable to extinction training for preventing the expression of fear memories. This hypothesis is supported by recent findings showing that extinction training within but not outside a reconsolidation time window permanently attenuates fear memories (Monfils et al, 2009; Schiller et al, 2010).

Theoretically, reconsolidation and extinction occur under similar conditions after memory retrieval. However, these two processes have distinct temporal and biochemical signatures (Suzuki et al, 2004; Schiller and Johansen, 2009; Makkar et al, 2010). Recent studies have shown that brief or weak re-exposure to a conditioned cue leads to reconsolidation of memories whereas more prolonged or repeated reexposure results in extinction of memories (Pérez-Cuesta and Maldonado, 2009). In addition, the $\alpha$-amino-3-hydroxyl-5-methyl-4-isoxazole-propionate receptor potentiator, 4-[2-(phenylsulfonylamino)ethylthio]-2,6-difluorophenoxy acetamide, has been reported to facilitate extinction of contextual fear without influencing reconsolidation processes (Yamada et al, 2009). These findings suggest that reconsolidation and extinction are two separate processes that can coexist after non-reinforced presentations of the conditioned cues (Lee et al, 2006). Although both strength- ening extinction training and disrupting reconsolidation process are reported to effectively reduce the emergence of learned conditioned responses, the effectiveness of facilitating extinction training and disrupting reconsolidation process is somewhat different. The predominant view is that extinction mechanisms generate further inhibitory association between the CS and the US, which actively counteract the tendency for the CS to activate the US (Likhtik et al, 2008; Ehrlich et al, 2009). As the inhibitor of association at the basis of extinction is generally more labile than its original stimulus, the previously extinguished fear tends to return with the passage of time or change in response to the environmental context (Norrholm et al, 2006). In addition, several recent studies have found that disrupting reconsolidation process may erase learned fear responses and prevent the return of fear (Duvarci and Nader, 2004; Eisenhardt and Menzel, 2007). Together, these results strongly suggest that the disruption of reconsolidation process can be used to eliminate fear memories.

It is widely accepted that extinction training involves the formation of new inhibitory memories rather than the forgetting or unlearning of memories (Myers and Davis, 2007). Since the extinction process does not eliminate the existing fear memories, fear responses often return over time. One of the major reasons for the lack of effectiveness of extinction training is that the extinction is a highly context-dependent process. On the other hand, the extinction is cue-specific, meaning that it does not generalize to other cues that have been fear conditioned but not extinguished (Davis et al, 2006). In this study, we have, in addition, extended these findings by demonstrating that although administration of DCS before extinction training strengthens extinction learning but it does not make this new learning any less dependent on the conditioned cues. The conditioned responses to the other cues previously paired remained unchanged. These observations are consistent with previous findings showing that DCS strengthens extinction of fear but does not eliminate context-specific renewal of the conditioned responses (Woods and Bouton, 2006). However, a recent study demonstrated that DCS can effectively reduce the context dependency of Pavlovian extinction of cocaine cues through action in the nucleus accumbens core (Torregrossa et al, 2010). Thus far, no other studies have shown that DCS can attenuate cueinduced reinstatement of responding by a reduction in the context dependency of extinction. It is therefore possible that the ability of DCS to reduce the context dependency of extinction is specific to cocaine-associated stimuli.

In contrast to the cue specificity of fear extinction training, the post-reactivation disruption of reconsolidation process in the DH but not in the BLA eliminates all learned associations between conditioned cues with the established fear memories. We demonstrate that the inhibition of $\mathrm{DH}$ protein synthesis or genetic suppression of CREB function after re-exposure to one cue previously paired disrupts both reconsolidation process and subsequent expression of fear responses to other conditioned cues, indicating that disrupting reconsolidation process in the $\mathrm{DH}$ could be a more effective strategy for clinically treating fear memories. However, our findings are not consistent with a recent report, showing that blocking protein synthesis or genetic suppression of CREB function in either the hippocampus or 
the amygdala blocked the reconsolidation of contextual fear memories (Mamiya et al, 2009). The simplest explanation for this apparent inconsistency could be the use of different fear conditioning paradigms (single cue $v s$ multiple cues), resulting in differences in brain areas and mechanisms recruited during memory reactivation. Hence, it seems likely that the DH and the BLA may perform different functions during the reconsolidation of contextual fear memories depending on the complexity of conditioning paradigms. What mechanism in the $\mathrm{DH}$ is responsible for the retrieval of fear memories and hence the requirement of protein synthesis for reconsolidation? A recent study has indicated that synaptic protein degradation is activated after memory retrieval and that this process is critical for destabilization of the pre-existing fear memories (Lee et al, 2008). In addition, the reconsolidation process requires de novo protein synthesis to restablize memory trace (Lee, 2008). Recently, it has been reported that the NMDA receptors, L-type voltage-gated calcium channels or cannabinoid CB1 receptors may be upstream signaling molecules involved in ubiquitin/proteasome-dependent synaptic protein degradation and may trigger the destabilization of reactivated fear memories (Mamou et al, 2006; Suzuki et al, 2008). Thus, these retrieval-induced destabilization processes may also trigger de novo protein synthesis for memory reconsolidation. Further investigation is required to validate this possibility.

Importantly, we identify distinct role of the $\mathrm{DH}$ and the BLA in processing fear memories. Our data indicate that the BLA has a significant role in the cue-specific reconsolidation of fear memories, whereas the DH is implicated in the post-reactivation reconsolidation of associative memory traces. Although results from previous studies have suggested that the hippocampus is involved in memory reconsolidation (Kida et al, 2002; Debiec et al, 2002), few studies have assessed how the hippocampus functions as a circuit implicated in reconsolidation process after memory retrieval. The traditional consolidation theory predicts that the hippocampus has only a temporary role in memory storage, after which time memories become independent of the hippocampus and are stored in the neocortex (Squire and Alvarez, 1995; Anagnostaras et al, 2001). Interestingly, there is some evidence that reactivation of a hippocampusindependent remote memory causes it to return to a hippocampus-dependent state again (Debiec et al, 2002). Our observations that blocking protein synthesis or CREB function in the DH 7 days after conditioning training comprehensively diminished the learned connections between fear responses and all paired environmental cues are consistent with the systems reconsolidation view that reactivation of a remote memory returns the memory traces to being hippocampus dependent (Debiec et al, 2002).

Memory retrieval usually takes place when only subsets of initial encountered cues are present. This task is known as pattern completion in which the brain reconstructs and recalls entire memory patterns from partial external cues or self-initiated internal processes and is believed to occur in the hippocampus (Leutgeb and Leutgeb, 2007; Fellini et al, 2009). Although the proposed ultimate storage site for longterm memory is the neocortex, results from previous studies have suggested that the hippocampus may serve as a memory coordinator for the retrieval of information stored at several neocortical sites (Small et al, 2001; Runyan and Dashm, 2005). The results of the present study not only support a crucial role of the DH in coordinating memory retrieval, but also provide the first experimental evidence that inhibition of DH protein synthesis after memory recall by exposure to only subsets of initial cues can lead to complete loss of the ability to retrieve memory by all of the previously encountered cues.

Results from previous studies have suggested that the amnesic effects produced by disrupting reconsolidation process could be transient (Lattal and Abel, 2004; Power et al, 2006; Prado-Alcala et al, 2006) or persistent (Debiec et al, 2002; Debiec and LeDoux, 2004; Duvarci and Nader, 2004; Boccia et al, 2005; Rossato et al, 2006). These seemingly inconsistent results may be related to the differential potency of interventions. The temporary amnesic effect induced by protein synthesis inhibitors may be related to insufficient degree or duration of protein synthesis inhibition (Alberini et al, 2006). In fact, stronger memories require longer (stronger) inhibition of protein synthesis to be permanently disrupted (Davis and Rosenzweig, 1978). We found that cycloheximide injections into the $\mathrm{DH}$ blocked the reconsolidation of reactivated fear memories over 1 month period, indicating that disrupting reconsolidation process by post-reactivation administration of protein synthesis inhibitors is long lasting. The absence of spontaneous recovery was not attributable to permanent damage to the $\mathrm{DH}$ in the cycloheximide-treated animals because they could be retrained (data not shown). In addition, we found that post-reactivation injections of the same dose of cycloheximide into the BLA are ineffective to disrupt the subsequent reactivation of fear memories. Likewise, overexpression of KCREB in the DH or BLA does not affect the animals to re-learn the contextual fear but impairs the consolidation of newly acquired fear memories. Therefore, the most parsimonious interpretation of these data is that post-reactivation interventions of reconsolidation process in the $\mathrm{DH}$ may induce a persistent inhibition in retrieval of fear memories.

Fear memories are often linked to multiple environmental cues and these interconnected cues may act as reminders of stressful events. The present study, using a protocol of mental conditioning paired with multiple aversive cues, found that extinction training is cue-specific. The conditioned responses to other cues remained unchanged. Given that it is often not clinically possible to inhibit the response to all previously reinforced cues, strengthening extinction to eliminate fear memories seems to be a limited approach. The other major challenge of extinction training-based exposure therapy is overcoming 'cognitive avoidance,' which is avoidance of anything that is a possible reminder of traumatic experience and could be shared with psychotherapists. Accordingly, these interconnected fear-related or aversive cues might not be consistently remembered during exposure therapy (Harvey et al, 2003).

In conclusion, our results provide strong evidence that although both disrupting reconsolidation process and strengthening extinction training are effective ways to reduce the emergence of conditioned fear responses, disrupting reconsolidation process in the $\mathrm{DH}$ is the only way to elicit generalization of fear inhibition. Our results, 
along with others (Kindt et al, 2009; Monfils et al, 2009; Schiller et al, 2010) may have important clinical implications for both the prevention and treatment of persistent and self-perpetuating memories in individuals suffering from PTSD or other anxiety disorders.

\section{ACKNOWLEDGEMENTS}

We thank the members of the Hsu's laboratory and $\mathrm{Dr}$ David Diamond for helpful discussion and suggestions. This work was supported by research grants from the National Science Council (NSC99-2321-B-006-001) and the National Health Research Institute (NHRI-EX99-9618NI), Taiwan.

\section{DISCLOSURE}

The authors declare no actual or potential conflict of interest. The authors affirm that there are no financial, personal, or other relationships with other people or organizations that have inappropriately influenced or biased their work.

\section{REFERENCES}

Alberini CM, Milekic MH, Tronel S (2006). Mechanisms of memory stabilization and de-stabilization. Cell Mol Life Sci 63: 999-1008.

Anagnostaras SG, Gale GD, Fanselow MS (2001). Hippocampus and contextual fear conditioning: recent controversies and advances. Hippocampus 11: 8-17.

Beiderbeck DI, Neumann ID, Veenema AH (2007). Differences in intermale aggression are accompanied by opposite vasopressin release patterns within the septum in rats bred for low and high anxiety. Eur J Neurosci 26: 3597-3605.

Boccia MM, Blake MG, Acosta GB, Baratti CM (2005). Memory consolidation and reconsolidation of an inhibitory avoidance task in mice: effects of a new different learning task. Neuroscience 135: 19-29.

Bouton ME (2002). Context, ambiguity, and unlearning: sources of relapse after behavioral extinction. Biol Psychiatry 52: 976-986.

Bouton ME, King DA (1983). Contextual control of the extinction of conditioned fear: tests for the associative value of the context. J Exp Psychol Anim Behav Process 9: 248-265.

Chang YJ, Yang CH, Liang YC, Yeh CM, Huang CC, Hsu KS (2009). Estrogen modulates sexually dimorphic contextual fear extinction in rats through estrogen receptor $\beta$. Hippocampus 19: $1142-1150$.

Davis HP, Rosenzweig MR (1978). Recovery as a function of the degree of amnesia due to protein synthesis inhibition. Pharmacol Biochem Behav 8: 701-710.

Davis M, Barad M, Otto M, Southwick S (2006). Combining pharmacotherapy with cognitive behavioral therapy: traditional and new approaches. J Trauma Stress 19: 571-581.

Debiec J, Ledoux JE (2004). Disruption of reconsolidation but not consolidation of auditory fear conditioning by noradrenergic blockade in the amygdala. Neuroscience 129: 267-272.

Debiec J, LeDoux JE, Nader K (2002). Cellular and systems reconsolidation in the hippocampus. Neuron 36: 527-538.

Dirikx T, Hermans D, Vansteenwegen D, Baeyens F, Eelen P (2004). Reinstatement of extinguished conditioned responses and negative stimulus valence as a pathway to return of fear in humans. Learn Mem 11: 549-554.

Duvarci S, Nader K (2004). Characterization of fear memory reconsolidation. J Neurosci 24: 9269-9275.
Effting M, Kindt M (2007). Contextual control of human fear associations in a renewal paradigm. Behav Res Ther 45: 2002-2018.

Ehrlich I, Humeau Y, Grenier F, Ciocchi S, Herry C, Luthi A (2009). Amygdala inhibitory circuits and the control of fear memory. Neuron 62: 757-771.

Eisenhardt D, Menzel R (2007). Extinction learning, reconsolidation and the internal reinforcement hypothesis. Neurobiol Learn Mem 87: 167-173.

Fanselow MS, LeDoux JE (1999). Why we think plasticity underlying Pavlovian fear conditioning occurs in the basolateral amygdala. Neuron 23: 229-232.

Fellini L, Florian C, Courtey J, Roullet P (2009). Pharmacological intervention of hippocampal CA3 NMDA receptors impairs acquisition and long-term memory retrieval of spatial pattern completion task. Learn Mem 16: 387-394.

Harvey AG, Bryant RA, Tarrier N (2003). Cognitive behaviour therapy for posttraumatic stress disorder. Clin Psychol Rev 23: 501-522.

Hermans D, Craske MG, Mineka S, Lovibond PF (2006). Extinction in human fear conditioning. Biol Psychiatry 60: 361-368.

Kearns DN, Weiss SJ (2007). Contextual renewal of cocaine seeking in rats and its attenuation by the conditioned effects of an alternative reinforcer. Drug Alcohol Depend 90: 193-202.

Kelly A, Laroche S, Davis S (2003). Activation of mitogen-activated protein kinase/extracellular signal-regulated kinase in hippocampal circuitry is required for consolidation and reconsolidation of recognition memory. J Neurosci 23: 5354-5360.

Kida S, Josselyn SA, Pena de Ortiz S, Kogan JH, Chevere I, Masushige $S$ et al (2002). CREB required for the stability of new and reactivated fear memories. Nat Neurosci 5: 348-355.

Kindt M, Soeter M, Vervliet B (2009). Beyond extinction: erasing human fear responses and preventing the return of fear. Nat Neurosci 12: 256-258.

Korzus E, Rosenfeld MG, Mayford M (2004). CBP histone acetyltransferase activity is a critical component of memory consolidation. Neuron 42: 961-972.

Kushner MG, Kim SW, Donahue C, Thuras P, Adson D, Kotlyar M et al (2007). D-cycloserine augmented exposure therapy for obsessive-compulsive disorder. Biol Psychiatry 62: 835-838.

Kutner RH, Zhang XY, Reiser J (2009). Production, concentration and titration of pseudotyped HIV-1-based lentiviral vectors. Nat Protoc 4: 495-505.

Lattal KM, Abel T (2004). Behavioral impairments caused by injections of the protein synthesis inhibitor anisomycin after contextual retrieval reverse with time. Proc Natl Acad Sci USA 101: 4667-4672.

Ledgerwood L, Richardson R, Cranney J (2005). D-cycloserine facilitates extinction of learned fear: effects on reacquisition and generalized extinction. Biol Psychiatry 57: 841-847.

Lee JL (2008). Memory reconsolidation mediates the strengthening of memories by additional learning. Nat Neurosci 11: 1264-1266.

Lee JL, Milton AL, Everitt BJ (2006). Reconsolidation and extinction of conditioned fear: inhibition and potentiation. J Neurosci 26: 10051-10056.

Lee SH, Choi JH, Lee N, Lee HR, Kim JI, Yu NK et al (2008). Synaptic protein degradation underlies destabilization of retrieved fear memory. Science 319: 1253-1256.

Leutgeb S, Leutgeb JK (2007). Pattern separation, pattern completion, and new neuronal codes within a continuous CA3 map. Learn Mem 14: 745-757.

Likhtik E, Popa D, Apergis-Schoute J, Fidacaro GA, Pare D (2008). Amygdala intercalated neurons are required for expression of fear extinction. Nature 454: 642-645.

Makkar SR, Zhang SQ, Cranney J (2010). Behavioral and neural analysis of GABA in the acquisition, consolidation, reconsolidation, and extinction of fear memory. Neuropsychopharmacology 35: $1625-1652$. 
Mamiya N, Fukushima H, Suzuki A, Matsuyama Z, Homma S, Frankland PW et al (2009). Brain region-specific gene expression activation required for reconsolidation and extinction of contextual fear memory. J Neurosci 29: 402-413.

Mamou C, Gamache K, Nader K (2006). NMDA receptors are critical for unleashing consolidated auditory fear memories. Nat Neurosci 9: 1237-1239.

Monfils MH, Cowansage KK, Klann E, LeDoux JE (2009). Extinction-reconsolidation boundaries: key to persistent attenuation of fear memories. Science 324: 951-955.

Myers KM, Davis M (2007). Mechanisms of fear extinction. Mol Psychiatry 12: 120-150.

Nader K, Hardt O (2009). A single standard for memory: the case for reconsolidation. Nat Rev Neurosci 10: 224-234.

Nader K, Schafe GE, Le Doux JE (2000a). Fear memories require protein synthesis in the amygdala for reconsolidation after retrieval. Nature 406: 722-726.

Nader K, Schafe GE, LeDoux JE (2000b). The labile nature of consolidation theory. Nat Rev Neurosci 1: 216-219.

Norrholm SD, Jovanovic T, Vervliet B, Myers KM, Davis M, Rothbaum BO et al (2006). Conditioned fear extinction and reinstatement in a human fear-potentiated startle paradigm. Learn Mem 13: 681-685.

Pavlov IP (1927). Conditioned Reflexes; an Investigation of the Physiological Activity of the Cerebral Cortex. Oxford University Press/Humphrey Milford: London.

Paxinos G, Watson C (2007). The Rat Brain in Stereotaxic Coordinates, 6th edn. Elsevier: Amsterdam; Boston.

Pérez-Cuesta LM, Maldonado H (2009). Memory reconsolidation and extinction in the crab: mutual exclusion or coexistence? Learn Mem 16: 714-721.

Power AE, Berlau DJ, McGaugh JL, Steward O (2006). Anisomycin infused into the hippocampus fails to block 'reconsolidation' but impairs extinction: the role of re-exposure duration. Learn Mem 13: 27-34.

Prado-Alcala RA, Diaz del Guante MA, Garin-Aguilar ME, DiazTrujillo A, Quirarte GL, McGaugh JL (2006). Amygdala or hippocampus inactivation after retrieval induces temporary memory deficit. Neurobiol Learn Mem 86: 144-149.

Radulovic J, Kammermeier J, Spiess J (1998). Relationship between fos production and classical fear conditioning: effects of novelty, latent inhibition, and unconditioned stimulus preexposure. J Neurosci 18: 7452-7461.

Radulovic J, Tronson NC (2010). Molecular specificity of multiple hippocampal processes governing fear extinction. Rev Neurosci 21: 1-17.

Rescorla RA, Heth CD (1975). Reinstatement of fear to an extinguished conditioned stimulus. J Exp Psychol Anim Behav Process 1: 88-96.

Ressler KJ, Mayberg HS (2007). Targeting abnormal neural circuits in mood and anxiety disorders: from the laboratory to the clinic. Nat Neurosci 10: 1116-1124.

Ressler KJ, Rothbaum BO, Tannenbaum L, Anderson P, Graap K, Zimand E et al (2004). Cognitive enhancers as adjuncts to psychotherapy: use of D-cycloserine in phobic individuals to facilitate extinction of fear. Arch Gen Psychiatry 61: 1136-1144.

Rossato JI, Bevilaqua LR, Medina JH, Izquierdo I, Cammarota M (2006). Retrieval induces hippocampaldependent reconsolidation of spatial memory. Learn Mem 13: 431-440.
Runyan JD, Dash PK (2005). Inhibition of hippocampal protein synthesis following recall disrupts expression of episodic-like memory in trace conditioning. Hippocampus 15: 333-339.

Schafe GE, Nader K, Blair HT, LeDoux JE (2001). Memory consolidation of Pavlovian fear conditioning: a cellular and molecular perspective. Trends Neurosci 24: 540-546.

Schiller D, Cain CK, Curley NG, Schwartz JS, Stern SA, Ledoux JE et al (2008). Evidence for recovery of fear following immediate extinction in rats and humans. Learn Mem 15: 394-402.

Schiller D, Johansen J (2009). Prelimbic prefrontal neurons drive fear expression: a clue for extinction-reconsolidation interactions. J Neurosci 29: 13432-13434.

Schiller D, Monfils MH, Raio CM, Johnson DC, Ledoux JE, Phelps EA (2010). Preventing the return of fear in humans using reconsolidation update mechanisms. Nature 463: 49-53.

Small SA, Nava AS, Perera GM, DeLaPaz R, Mayeux R, Stern Y (2001). Circuit mechanisms underlying memory encoding and retrieval in the long axis of the hippocampal formation. Nat Neurosci 4: 442-449.

Squire LR, Alvarez P (1995). Retrograde amnesia and memory consolidation: a neurobiological perspective. Curr Opin Neurobiol 5: 169-177.

Stein DJ, Matsunaga H (2006). Specific phobia: a disorder of fear conditioning and extinction. CNS Spectr 11: 248-251.

Suzuki A, Josselyn SA, Frankland PW, Masushige S, Silva AJ, Kida S (2004). Memory reconsolidation and extinction have distinct temporal and biochemical signatures. J Neurosci 24: 4787-4795.

Suzuki A, Mukawa T, Tsukagoshi A, Frankland PW, Kida S (2008). Activation of LVGCCs and CB1 receptors required for destabilization of reactivated contextual fear memories. Learn Mem 15: 426-433.

Torregrossa MM, Sanchez H, Taylor JR (2010). D-cycloserine reduces the context specificity of pavlovian extinction of cocaine cues through actions in the nucleus accumbens. J Neurosci 30: 10526-10533.

Tronson NC, Taylor JR (2007). Molecular mechanisms of memory reconsolidation. Nat Rev Neurosci 8: 262-275.

Vansteenwegen D, Vervliet B, Hermans D, Beckers T, Baeyens F, Eelen P (2006). Stronger renewal in human fear conditioning when tested with an acquisition retrieval cue than with an extinction retrieval cue. Behav Res Ther 44: 1717-1725.

Walker DL, Ressler KJ, Lu KT, Davis M (2002). Facilitation of conditioned fear extinction by systemic administration or intraamygdala infusions of $\mathrm{D}$-cycloserine as assessed with fearpotentiated startle in rats. J Neurosci 22: 2343-2351.

Walton KM, Rehfuss RP, Chrivia JC, Lochner JE, Goodman RH (1992). A dominant repressor of cyclic adenosine $3^{\prime}, 5^{\prime}$-monophosphate (cAMP)-regulated enhancer-binding protein activity inhibits the cAMP-mediated induction of the somatostatin promoter in vivo. Mol Endocrinol 6: 647-655.

Woods AM, Bouton ME (2006). D-cycloserine facilitates extinction but does not eliminate renewal of the conditioned emotional response. Behav Neurosci 120: 1159-1162.

Yamada D, Zushida K, Wada K, Sekiguchi M (2009). Pharmacological discrimination of extinction and reconsolidation of contextual fear memory by a potentiator of AMPA receptors. Neuropsychopharmacology 34: 2574-2584.

Yang CH, Huang CC, Hsu KS (2004). Behavioral stress modifies hippocampal synaptic plasticity through corticosterone-induced sustained extracellular signal-regulated kinase/mitogen-activated protein kinase activation. J Neurosci 24: 11029-11034.

Supplementary Information accompanies the paper on the Neuropsychopharmacology website (http://www.nature.com/npp) 